Heinz Steinbring

\title{
Mathematische Begriffe in didaktischen Situationen: das Beispiel der Wahrscheinlichkeit
}

In: Journal für Mathematik-Didaktik, Jg. 6 (1985), H. 2

S. 85-118 


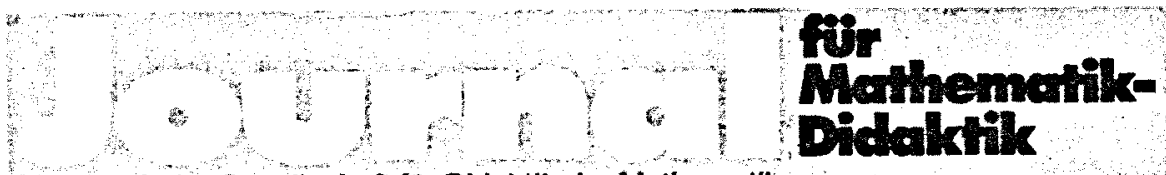

\section{Zeitschrift der Gesellschaft für Didaktik der Mathematik}

Jahrgang 6 (1985) Heft 2

Ferdinand Schöningh · Paderborn

Das JMD ist eine internationale wissenschaftliche Zeitschrift, in der Originalbeiträge aus allen Bereichen mathematikdidaktischer Forschungs- und Entwicklungsarbeit erscheinen. Es besteht Offenheit gegenüber Bezugswissenschaften (wie Pädagogik, Psychologie, Soziologie oder Philosophie) und gegenüber Nachbarwissenschaften (wie Didaktik der Physik oder Sprachdidaktik). Die Beiträge sollen jedoch in jedem Fall das Lernen und Lehren von Mathematik betreffen.

JMD wird von der Gesellschaft für Didaktik der Mathematik herausgegeben. Die Leitung obliegt einem Herausgebergremium, das durch ein wissenschaftliches Beratungskomitee unterstützt wird. Die Herausgeber entscheiden über eine (ggf. bedingte) Annahme oder Ablehnung eingereichter Manuskripte, i. a. mit Hilfe zweier Gutachter.

\section{Herausgebergremium}

Werner BLUM

Gesamthochschule

Universität Kassel

Fachbereich Mathematik

Heinrich - Plett - Str. 40

D-3500 Kassel
Wiltibald DÖRFLER

Universität für

Bildungswissenschaften

Institut für Mathematik

Universitätsstr. $65-67$

A-9010 Klagenfurt
Hans SCHUPP

Universität

des Saarlandes

Fachbereich Mathematik

St. Johanner Stadtwald

D-6600 Saarbrücken

\section{Wissenschaftliches Beratungskomitee}

Jürgen DIEDERICH. Frankfurt

Roland FISCHER, Klagenfurt

Hans - Niels JAHNKE, Bielefeld

Arnold KIRSCH. Kassel

Gustav Adolf LÖRCHER, Freiburg

Günter PICKERT, Gießen

\author{
Siegbert SCHMIDT, Köln \\ Hans-Georg STEINER, Bielefeld \\ Ursula VIET, Osnabrück \\ Hans-Joachim VOLLRATH, Würzburg \\ Gerd WALTHER, Kiel \\ Heinrich WINTER, Aachen
}

JMD erscheint in 4 Heften pro Jahr. Mitglieder der GDM erhalten die Zeitschrift kostenlos. Der Preis im Buchhandel beträgt je Heft DM 10,-. Sonderhefte kosten für Mitglieder der GDM DM 7,-, für Nichtmitglieder DM 14,--

\section{Hinweise für Autoren}

Manuskripte in deutscher, englischer oder französischer Sprache sind - zusammen mit einem Abstract in deutsch - maschinengeschrieben und in fünf Exemplaren an einen der Herausgeber zu senden. Bei empirischen Arbeiten und Projekt beschreibungen, bei denen für die Nachvollziehbarkeit ausführliche Berichte und Ver suchsmaterialien vonnöten sind, sollten diese der Redaktion auf Anforderung zur Ver fügung stehen sowie vom Leser beim Autor (gegen Erstattung der Kopierkosten) an gefordert werden können. Im Falle der Annahme der Arbeit ist vom Autor nach den Richtlinien des Verlags auf speziellem Papier ein Typoskript herzustellen. Der Autor erhält 50 Sonderdrucke kostenlos.

\footnotetext{
- 1985 by Ferdinand Schöningh al Paderborn Printed in Germany
}

Alle Rechte auch die des auszugswe'sen Nachdrucks der totomechanischen Wiedergabe und der Uberset. zung vorbehatten. Dies betrift auch de Verveltaltigungund Ubentragung einzelner Textabschnitte. Zeichnungen oder Bilder durch alle Verahren we Specherung und Ubertragung auf Papier. Transparente. Filme. Bänder. Flatten und andere Medien. sowet es nicht $\$ \$ 53$ und 54 URG ausdrucklich gestatten

ISSN 0173-5322

Anzeigenverwaltung: Ferdnand Schoningh Paderborn 


\section{H. Steinbring}

\section{Mathematische Begriffe in didaktischen Situationen: Das Beispiel der Wahrscheinlichkeit ${ }^{\star}$}

Summary: The article demonstrates the importance of an autonomous epistemology of school mathematics (in comparison with scientific mathematics). This is especially examplified with respect to the concept of probability. The understanding and the learning of mathematical concepts are closely connected with the available means of representation (of the mathematical knowledge) and the means of activity (of the pupils).

\section{Einleitung}

"The real problem which confronts mathematics teaching is not that of rigour, but the problem of the development of 'meaning', of the 'existence' of mathematical objects." (THOM, S.202) Die Schwierigkeiten, diese bekannte und weitgehend akzeptierte Forderung René THOMs angemessen zu erfüllen, sind vor allem darin begründet, daß im Mathematikunterricht "Begriffsbedeutung" und "Existenz mathematischer Gegenstände" nicht disziplinär vorgegeben werden können, sondern durch vielfältige soziale und gegenständliche Aspekte im Unterrichtsprozeß erst konstituiert werden. Auf seiten der Wissenschaft versucht man, dieser Situation durch interdisziplinäre Ansätze zu begegnen; auf seiten der Schulpraxis werden zunehmend soziale Rahmungen und inhaltliche Auffassungen in realen Unterrichtsprozessen erforscht.

Eine systemtheoretisch orientierte Charakterisierung der Mathematikdidaktik als einer komplexen Grundlagenwissenschaft steht vor folgender Aufgabe: Es gilt, die einzelnen Komponenten dieses Systems zu untersuchen und dabei gleichzeitig die Untersuchung unter der Perspektive des Gesamtsystems vorzunehmen, d.h. immer auch die Beziehungen der Systemkomponenten zueinander im Auge zu behalten. Was sind mögliche Komponenten des Systems "Mathematikdidaktik"? Im Sinne einer Definition der Mathematikdidaktik als einer angewandten Grundlagenwissenschaft kann man drei Hauptkomponenten identifizieren: Forschung, Entwicklung und Praxis (vgl. u.a. AG MATHEMATIKLEHRERBILDUNG 1981, STEINER 1984).

Für jedes dieser drei Subsysteme hat man in unterschiedlicher Form den interdisziplinären Charakter der Mathematikdidaktik zu berücksichtigen. Die Vielfalt ihrer interdisziplinären Bezüge ist bekannt: Pädagogik, Psychologie, Sozialwissenschaften, Philosophie, Wissenschaftstheorie, Geschichte und natürlich die

* Ausarbeitung eines Vortrags, gehalten am 25.8.84 auf dem 5. Internationalen Kongre $B$ für Mathematikunterricht in Adelaide $(24 .-30.8 .84)$, in der Study Group "Theory of Mathematics Education (TME)". 
Mathematik selbst, um nur die wichtigsten zu nennen. Ob im Bereich der Forschung, der Materialentwicklung oder der Schulpraxis, diese interdisziplinären Systemelemente der Mathematikdidaktik und ihr Zusammenwirken sind in je unterschiedlicher Form fundamental für jegliches mathematikdidaktisches Arbeiten.

"Ein systemtheoretisches Herangehen an den Gegenstand der Fachdidaktik ... muß meines. Erachtens von der zentralen Rolle der Grundwissenschaft, also in unserem Falle der Mathematik, für alle anderen Systemelemente ausgehen. Was die Fachdidaktik von der Grundlagenwissenschaft unterscheidet, ist der spezifische Charakter des Verhältnisses von inhaltlichen und sozialen Bestimmungsmomenten."

(OTTE 1982a, S.9) Versteht man diese Aussage ausschließ1ich dahingehend, da $B$ man in der Mathematikdidaktik die (Schul-) Mathematik nicht außer acht lassen darf, so wird sie (scheinbar) trivial. Es gibt jedoch auch sog. inhaltsneutrale didaktische Forschungsrichtungen bzw. Ansätze, die bewußt neue disziplinäre Forschungsmethodologien auf didaktische Probleme anwenden und ausarbeiten, ohne damit unmittelbar inhaltliche Bedingungen bzw. Ansprüche erfüllen zu wollen.

Der Kern der These von der zentralen Rolle der Mathematik besteht darin, da $\beta$ im Rahmen der Systembeziehungen der Fachinhalt nicht von außen vorgegeben, daB Schulmathematik kein "Fertigprodukt" der Fachwissenschaft sein kann, sondern daß sich Bedeutungen und Existenz der Gegenstände (THOM) der Schulmathematik im Gesamtsystem "Mathematikdidaktik" herausbilden sowie im Unterrichtsprozeß spezifische Ausprägungen erfahren - wobei die Mathematik das ausgezeichnete Systemelement darstellt.

Diese These wird nur sinnvoll, wenn der Fachinhalt nicht einfach als eine dem System äußerliche Struktur betrachtet wird; das mathematische Wissen muß zu einem integralen, "variablen" Systemelement werden. Die Schulmathematik muß in grundlegender Weise vielfältige Bezüge zu den anderen Systemelementen aufnehmen und reflektieren. Verglichen mit der wissenschaftlichen Mathematik werden sich dabei Anderungen, Abweichungen, neuartige Bedeutungen sowie die Betonung besonderer Arbeits- und Interpretationsweisen für die Schulmathematik ergeben. In gewisser Weise wird Schulmathematik so zu einer "anderen Mathematik". Dieses Anliegen kann man als Ausdruck der THOMschen Forderung ansehen, wie es auch von vielen Didaktikern bearbeitet wird. Vor allem Untersuchungen zur Begriffsinterpretation in der Schulmathematik setzen sich beispielhaft mit dieser Problematik auseinander (vgl. u.a. DORFLER 1984b, LEPPIG 1985, VOLLRATH 1984, WINTER 1983b).

Die folgende Analyse versteht sich als Beitrag zur didaktischen Diskussion um den besonderen Charakter mathematischer Begriffe sowie zur Auffassung der Schulmathematik. Dabei soll vor allem die Bedeutung des Gegenstandes der (Schul-) 
Mathematik sowie der Mittel der Darstellung und der Tätigkeit beisnielhaft herausgearbeitet werden, deren Funktion für das Begriffslernen häufig implizit bleibt und bisher noch wenig hervorgehoben wurde (siehe z.B. BIEHLER 1984, FISCHER 1984, JAHNKE 1984).

Dies zu verdeutlichen, erscheint uns der Wahrscheinlichkeitsbegriff besonders geeignet. Schon in der historischen Diskussion hat er eine wichtige Vorreiterrolle gespielt und z.B. dazu beigetragen, den modernen axiomatischen standpunkt zu Beginn des 20. Jahrhunderts durchzusetzen ( $\mathrm{vgl}$. FREUDENTHAL 1968, STEINBRING 1980a und 1980b). Auch für den Mathematikunterricht kann der Wahrscheinlichkeitsbegriff so etwas wie ein Prüfstein sein, der unterliegende Auffassungen zur Mathematik ans Licht bringt und alte Stoffbereiche neu beleuchtet. Der mathematische Begriff wird als die Keimzelle des mathematischen Inhalts aufgefaßt. Ganz im Sinne unserer systemtheoretischen Zugangsweise wird der Begriff als ein Subsystem im Gesamtsystem Mathematik verstanden. Diese Interpretation orientiert sich nicht nur an fachlichen Aspekten, sie versucht, die Vielfalt interdisziplinärer Bezïge mathematikdidaktischer Arbeit einzubeziehen, indem sie den mathematischen Begriff immer im Kontext didaktischer Situationen sieht.

Die folgenden Oberlegungen, insbesondere zur Rolle des Wahrscheinlichkeitsbegriffs, gründen sich u.a. auf systematische Unterrichtsbeobachtungen und Lehrerfortbildungen zur Stochastik (in der Sek.I), die im Rahmen des Forschungsprojekts LEDIS (Lehrplan und Differenzierung in der SI) am IDM/Bielefeld begleitend durchgeführt wurden.

\section{Zum Selbstverständnis der Schulmathematik}

"In fact, whether one wishes or not, all mathematical pedagogy, even if scarely coherent, rests on a philosophy of mathematics." (THOM 1973) Nicht nur in der Mathematikdidaktik, auch für den Unterricht selbst hat THOMs Feststellung Gültigkeit. Die Auffassung von Mathematik, die Lehrer mitbringen, entstammt der impliziten Philosophie von Mathematik, wie sie im Studium vertreten wird. Vorherrschend ist hier ein "genügend offener Platonismus" (GOODMAN 1979, S.23).

"Ich möchte rasch das Bild der mathematischen Tätigkeit zusammenfassen, das der Platonismus zeichnet. Nach dieser Ansicht steht der Mathematiker vor einer großen Vielzahl abstrakter Strukturen, die schon vor seiner mathematischen Tätigkeit vorhanden sind. Er schafft diese Strukturen nicht, er findet sie. Im Verlaufe seiner Ausbildung, und wenn er später seine Geisteskräfte entwickelt, gestaltet und verfeinert er seine Intuition über diese Strukturen. Typischerweise gewinnt er natürlich mehr Erkenntnisse über bestimmte Strukturen als über andere. Seine Intuition wird von den Wahrheiten über die mathematische Welt geformt, die von seinen 
Vorläufern und Kollegen entdeckt worden sind, und dann befähigt inn seine Intuition wiederum, neue Strukturen $z u$ finden und neue Annahmen über die Strukturen aufzustellen. Um diese Annahmen zu verifizieren, um die Fragen zu beantworten, auf die er stöBt, führt er Konstruktionen aus, entwickelt Argumentationen, definiert dann neue Begriffe. Diese Konstruktionen wiederum werden in mathematischem Deutsch ausgedrückt, durch Berechnungen gestützt, in strenge und formale Form gebracht. Dadurch werden sie öffentlich zugänglich und verifizierbar und werden Teil der gesellschaftlichen Gesamtdialektik, durch die sich die Mathematik entwickelt.

Dies scheint mir eine recht befriedigende Darstellung dessen, was der reine Mathematiker tut. Ich meine sogar, daß die meisten heutigen Mathematiker, wenn sie sich die Mühe gemacht haben, dies für sich selbst zu artikulieren, irgendeine variante dieser Ansicht akzeptieren würden. Der Platonismus ist so befriedigend, daß nur sehr wenige Mathematiker oder Mathematiktheoretiker in neuerer Zeit ein Bedürnis verspürt haben, darüber hinauszugehen." (GOODMAN, S.21)

Von einer solchen Auffassung ist die Schulmathematik wesentlich geprägt. Die mathematischen Begriffe des Curriculums (der Sek.I) bilden das fachliche Netzwerk des mathematischen Wissensbestandes, sie dienen der Analyse der "Vielzah1 abstrakter Strukturen" (GOODMAN). Tendenziell lassen sich hier zwei Grundtypen von Begriffen erkennen. Zum einen hat man ausgezeichnete fundamentale Begriffe, die sich durch das gesamte Curriculum hindurchziehen; hierzu gehören beispielsweise der Zahlbegriff, die geometrischen Begriffe der Ebene und des Raumes, der Funktionsbegriff, strukturelle Begriffe der Algebra oder auch der Wahrscheinlichkeitsbegriff. Zum anderen gibt es eine große Vielfalt bestimmter lokaler Spezifizierungen dieser Grundbegriffe, die häufig nur an wenigen Stellen des Curriculums erscheinen und meist auch nur in begrenztem Umfang ausgearbeitet werden. Hierzu lassen sich beispielsweise folgende Begriffe zählen: Binomische Formel, Schwerpunkt im Dreieck, Prisma, Median, Irrationale Zahl usw.

Diese Unterscheidung wird ähnlich in der didaktischen Literatur getroffen. Winter betont u.a. "Unterschiede in Rang und Reichweite" der Begriffe. "Lokal bedeutsame Begriffe ... bis hin zu weitreichenden, fundamental en Begriffen." (WINTER 1983b, S.181) Auch LEPPIG unterscheidet zwischen sog. Fundamentalbegriffen und abgeleiteten Begriffen. "Aus den Fundamentalbegriffen entstehen durch Zusammensetzen neue Begriffe, aus diesen wiederum neue. Zusammengesetzte Begriffe sind ein Begriffsschema aus den sie definierenden Begriffen." Und weiter unten:"Man kann daher die an einer neuen Begriffsbildung beteiligten Begriffe einfach als Menge von Begriffen schreiben und diese Menge die Begriffskette des neuen Begriffs nennen. Den neu definierten Begriff einen Kettenbegriff." (LEPPIG, S.68/9) 
Diese Auffassung bringt prägnant zum Ausdruck, daß die innere, logische Begriffsstruktur Richtschnur für Begriffsbegründungen ist, deren formale bzw. abstrakte Form zwar häufig kritisiert wird, die jedoch letztlich in dieser oder ähnlicher Weise die Argumentationsgrundlage bleibt. In diesem Rahmen können die abgeleiteten Begriffe zwar auf die Grundbegriffe zurückgeführt werden; für diese selbst ist eine solche Begründung nicht möglich. Es entsteht so etwas wie ein "Begründungsvakuum - eine Situation, in der die Mathematiker ohne systematische Erklärung des Charakters der Strukturen waren, mit denen sie sich befaßten." (GOODMAN, S.22) Auch Mathematiklehrer stehen häufig vor einem solchen Begründungsvakuum, wenn primär die strukturelle Systematik die Begründungen liefern so11, obwohl Bedeutungen nicht rein logisch, sondern nur aus eigenständigen "Gegegenständen" außerhalb des Strukturgefüges erwachsen.

Wenn auch in abgeschwächter Form, so hat sich die Schulmathematik doch im Prinzip gleichermaßen dem Problem des Begründungsvakuums gestellt:"Die axiomatische Mengentheorie beeilte sich, diese Lücke zu füllen. Die mengentheoretische Sicht der Grundlagen jedoch ist Platonismus in einer äußerst reduktionistischen Form. Alle objekte der Welt des Mengentheoretikers sind abstrakt. Selbst wenn Individuen zugestanden werden, und diese sind gewöhnlich ausgeschlossen, wird von diesen Individuen angenommen, $d a ß$ sie weder eine innere Struktur noch bewußte Beziehungen zueinander haben. Sie sind lediglich abstrakte Punkte. Folglich zieht eine Reduzierung der gesamten Mathematik auf die Mengentheorie eine Verengung des Gegenstandes der Mathematik nach sich, mit der jedwede konkrete Realität ausgeschlossen wird." (GOODMAN, S.22)

Wohlgemerkt, in der Schule wurde nie axiomatische Mengenlehre betrieben; man wollte sich jedoch in angemessen elementarisierter Form die Vorteile eines solchen strukturorientierten Ansatzes für eine durchgängige Begriffsbegründung zunutze machen. Mit dem Mengenbegriff sollte ein einziger Grundbegriff für die Schulmathematik geschaffen werden. Dieser Begriff schien unmittelbar verständlich und aus sich heraus plausibel zu sein und gleichzeitig als Grundlage für die definitorische Ableitung aller anderen mathematischen Begriffe dienen zu können. Im Prinzip war hiermit auch intendiert, die Begrifflichkeit der Schulmathematik eindeutig und streng zu fassen.

Nicht nur die Auswirkungen der sog. "New Math", die in expliziter Form zum Glück immer weniger im Unterricht vertreten wird, auch die impliziten philosophischen Standpunkte zur Schulmathematik heben letztlich die interne Konsistenz der Begriffsstruktur, den logischen Zusammenhang der Wissenselemente als entscheidend hervor. Und dabei spielt, ganz im Sinne des Zitats von THOM, die philosophische Orientierung eine wichtige Rolle - auch wenn im einzelnen im Mathematikunterricht 
kein expliziter mathematischer Formalismus oder eine axiomatische Mengenlehre betrieben werden konnte und wurde. So schränkt beispielsweise WINTER, der ausführlich die unterrichtlichen Besonderheiten des Beweisens und der Begriffsbildung diskutiert (vgl. WINTER 1983a und 1983b), die Auffassung von (Schul-) Mathematik mit der Aussage ein:"Mit diesen Bedenken gegen die verbreitete negative Bestimntheit der Beweisbedurftigkeit will ich natürlich nicht behaupten, daß die Mathematik so etwas wie eine empirische Wissenschaft sei." (WINTER 1983a, S.66) Wenn nicht durch einen quasi-empirischen Bezug zu Gegenständen außerhalb der mathematischen Strukturen, dann können Begründung und Bedeutung mathematischer Begriffe letztlich nur durch interne, strukturell-disziplinäre Beziehungen reguliert werden. Die Orientierung der Schulmathematik am Bild der wissenschaftlichen Mathematik sowie das Vorherrschen philosophischer Positionen zur reinen Mathematik wird auch aus anderen schulmathematischen Problemen ersichtlich.

So ist es beispielsweise gang und gäbe, die Verallgemeinerung eines mathematischen Begriffs oder die mathematische Abstraktion als das Weglassen von spezifischen Merkmalen zu verstehen. Diese Auffassung ist der Definition von Begriffen auf der Grundlage der Mengenlehre konform: Beispielsweise entsteht nach dieser Vorstellung der abstrakte Zahlbegriff dadurch, daB dem Kind eine Vielzahl von verschiedenen Mengen (von objekten) präsentiert wird, aus denen nach und nach durch Vernachlässigung überflüssiger Eigenschaften der abstrakte Begriff erwächst. In kritischer Gegenüberstellung wird mathematische Abstraktion jedoch nicht als Reduzierung von Eigenschaften, sondern umgekehrt als die Ausweitung von interpretativen und operativ anwendungsbezogenen Begriffsaspekten gesehen ( $\mathrm{vg}$ 1. hierzu JAHNKE 1984). SCHREIBER stellt z.B. der Begriffsbildung durch Abstraktion, der er eine Oberbetonung vorwirft, die Begriffsbildung durch Idealisierung gegenüber (SCHREIBER 1980). Diese ermöglicht es - so SCHREIBER -, die Bedingungen für Abweichungen zwischen (mathematischem) Modell und Wirklichkeit qualitativ zu bestimmen (SCHREIBER, S.55).

In ähnlicher Absicht kritisiert DORFLER, daB die Begriffsbildung durch Vergleich und Abstraktion so nicht zu relevanten Begriffen führt. "Man kann dazu anmerken, da $B$ es in einem solchen aristotelischen Begriffsbildungsmodell völlig unklar bleibt, wieso gerade dieses und nicht ein anderes Gemeinsames isoliert und zum Begriff gemacht wird. Nimmt man nicht eine metaphysische Fähigkeit des Menschen zur Erkenntnis des 'Wesentlichen' ... an, das in einer absoluten Ordnung gegeben ist, so ist eine derartige Begriffsbildung dem Zufall ausgesetzt oder zumindest der Willkür des Menschen." (DORFLER 1984b, S.245)

Ein zweiter Punkt, an dem die besondere Natur der Schulmathematik sichtbar wird, ist das Beweisen. Der Beweis wird als das Kriterium angeführt, das die Mathematik vom Rechnen unterscheidet. Häufig wird der Beweis ausschlieBlich entsprechend dem 
Bild, das man vom Beweisen in der reinen Mathematik hat, als eine logisch konsistente Ableitung eines mathematischen Sachverhaltes aus bekannten Zusammenhängen angesehen. In der Schulmathematik wird eine Perspektive vernachlässigt, in der das Beweisen als eine vielfältige, explorative Tätigkeit zur Auffindung und Entwicklung neuen Wissens verstanden wird (vgl. FISCHER, S.154; JAHNKE 1978). Beweisen in der Schulmathematik ist das Herstellen einer logischen Verbindung zwischen bekannten Tatsachen - zumindest sind diese dem Lehrer bekannt (und der Schüler glaubt diese auch ohne jeglichen Beweis). Auch dieser Beweisauffassung korrespondiert eine strukturmathematische Kodifizierung schulmathematischen Wissens.

Eine ähnliche Kritik formuliert WINTER. "Häufig wird betont: Durch einen deduktiven Beweis wird lediglich Wissen gesichert und nicht etwa vermehrt, denn in deduktiven Schlüssen kann die Konklusion nicht mehr Information enthalten als bereits in den Prämissen enthalten ist. Deduktionen stellen lediglich eine logische Hierarchisierung der Sätze dar, es handelt sich beim Deduzieren um das Aneinanderreihen von logischen Tautologien .... Wenn das Beweisen als lediglich wissenssichernd angesehen wird - und das scheint leider verbreitet der Fall zu sein -, so darf man sich über mangelnde Beweisfreudigkeit der Schüler nicht wundern. Beweise erscheinen dann nämlich als den eigentlichen, d.h. wissensvermehrenden Positionen nachgerückte Prozeduren, die allenfalls der Form halber noch notwendig sind oder das (aus unerfindlichen Gründen) schlechte Gewissen des Lehrers beruhigen." (WINTER 1983a, S.72/3)

Diese Oberlegungen verdeutlichen, daß sich die aktuelle Schulmathematik (der Sek.I) relativ stark an Auffassungen der reinen Mathematik orientiert. Schulmathematik ist so zu einem elementarisierten Typ von reiner Mathematik geworden. Dieser Anspruch wird durch die besonderen Bedingungen schulischen Lernens (sozialer, pädagogischer, psychologischer sowie unterrichtsorganisatorischer Art) relativiert, jedoch nicht grundsätzlich in Frage gestellt.

Die Herausbildung dieser Auffassung ist stark mit der fundamentalistischen Reforminitiative von BOURBAKI verbunden. Ihre pädagogische Wirksamkeit erhielt sie vor allem durch das Werk von BRUNER, Z.T. auch durch die Arbeiten von PIAGET (vgl. hierzu AG MATHEMATIKLEHRERBILDUNG, Kap.4). In einer kritischen Analyse einer solchen fundamentalistischen Interpretation von Schulmathematik zieht OTTE folgendes Resumee:"Fundamentalistische Initiativen betonen die Bedeutung der Struktur der Disziplin und der dafür notwendigen Grundbegriffe. Sie gehen aus von der Identität der Tätigkeiten des mathematischen Wissenschaftlers und des Schülers im Mathematikunterricht. ... Schulmathematik und wissenschaft 1 iche Mathematik sind nicht voneinander verschieden. Die letztere ist zwar etwas komplizierter, aber der Schulmathematik prinzipiell gleich. Beide sind bestimmt durch 
jene drei BOURBAKIschen Mutterstrukturen: Gruppe, Topologie und Ordnung. Die Schulmathematik legt die Fundamente für die darauf aufbauende Ausarbeitung der wissenschaftlichen Mathematik und findet in dieser Perspektive ihre tendenziell einzige Rechtfertigung." (OTTE 1982b, S.13/14)

Neben der fundamentalistischen Interpretation der Schulmathematik stellen die sog. instrumentalistischen Reforminitiativen die Besonderheiten von Schulmathematik in den Mittelpunkt, beinhalten jedoch die Gefahr einer obermethodisierung des Unterrichts. "Der zweite Typus von didaktischer Initiative der Mathematik ... sieht die Eigenständigkeit des didaktischen Problems wesentlich deutlicher. Ich möchte diesen Typus instrumentalistischen Typus nennen. Instrumental istische Initiativen betonen die Probleme der Explizierung und der Darstellung des Wissens als wesentlich. Sie betrachten die durch die Explizierungs- und Darstellungsmittel gegebenen Möglichkeiten der Stimulierung, Motivierung und Steuerung der Lerntätigkeit als entscheidend. Sie sind auch antifundamentalistisch in dem Sinn, daß sie Inkohärenz und Pluralismus akzeptieren. ...

Eine Schwierigkeit be i der Entwicklung einer 'instrumentalistischen' Konzeption (eine Schwierigkeit, die für viele sinnlose Methodisierungen verantwortlich ist) liegt darin, daB es auf der Ebene der Darstellung und der Darstellungsmittel keine eindeutige Grenze gibt zwischen psychologisch rein funktionalen oder rein konventionellen Zeichen, z.B. reinen Konzentrationshilfen und auf den Inhalt wesentlich bezogenen Zeichenmodellen. ...

Instrumentalistische Initiativen bergen, wenn sie nicht sorgfältig die verschiedenen Wissensformen ins Verhältnis setzen, die Gefahr in sich, den Zusammenhang Wissenschaft - Schule gänzlich zu negieren. Die dann gänzlich pädagogisch-methodisch ausgerichtete Argumentation bezüglich des Aufbaus von Lehrplan und Curriculum ist kaum objektivierbar und führt in der Regel zu zweifelhaften Ergebnissen." (OTTE 1982b, S.15/16)

In einer naiven Sicht der Mengenlehre lassen sich für den Unterricht beide Auffassungen integrieren, die fundamentalistische ebenso wie die instrumentalistische. Aus schulpraktischer Perspektive wird zudem deutlich, warum der Elementarismus, eine Spielart des Instrumentalismus, für den Mathematikunterricht scheinbar Vorteile bietet:"Es gibt einen weiteren Grund, warum der Elementarismus in der Schulmathematik eine so starke Stellung hat. Dieser Grund liegt darin, daB er die Kommunikation zwischen Lehrern und Schülern scheinbar eindeutig und unmißverständlich macht. In der elementaristischen Auffassung haben alle Begriffe ihre feste Bedeutung, es gibt scheinbar keine unterschiedlichen Bedeutungswahrnehmungen bei Lehrern und Schuilern. Die Dinge gehen alle ihren geordneten und vorgezeichneten Gang." (JAHNKE 1984, S.34)

Im Rahmen der Entwicklung begrifflichen Denkens formuliert WINTER eine ähnliche 
Kritik gegenüber zu starken methodischen Absicherungen. "Die Sorge um begriffliche Klarheit steht immer in der Gefahr, in Präzisionsfanatismus zu entarten. Dieser äußert sich u.a. erstens in einer demotivierenden Bereitstellungsbeflissenheit (zuerst einmal viele Definitionen und nur wenige Sätze, diese dann aber womöglich auch noch eher trivial), zweitens in der Neigung, auch das zu definieren, wovon gar kein systematischer Gebrauch gemacht wird, drittens in der Sucht, möglichst viele Fachtermini zu verwenden, und viertens in dem Bestreben, möglichst von Anfang an endgïltige, wasserdichte Formulierungen anzustreben." (WINTER 1983b, S.196/7)

Das Schwanken der Schulmathematik und didaktischer Positionen zwischen fundamentalistischen und instrumentalistischen Initiativen (bzw. Unterarten hiervon) ist kein neuartiges Phänomen; schon zu Beginn des 19. Jahrhunderts lassen sich im Kontext der Entwicklung des mathematischen Lehrplans der preußischen Gymnasien diese Standpunkte feststellen (vgl. JAHNKE 1982).

In seinem Bestreben, der wissenschaftlichen Mathematik nachzueifern, trifft der Unterricht auf grundlegende Probleme. Die tatsächliche Handhabung und Entwicklung von Begriffen in Wissenschaft und Schule zeigt Gegensätze, wie sie mit der Obernahme wissenschaftlich orientierter Mathematikauffassungen nicht beabsichtigt waren.

Für den Mathematikunterricht ist die vollständige und exakte Definition mathematischer Begriffe in einer prinzipiellen Weise der Endzustand des Lernprozesses. Der in der Definition präsentierte Begriff ist ein abgeschlossenes und lokal begrenztes Faktum. Es sind zwar pädagogisch geleitete und den Lernprozeß motivierende Hinführungen zum Begriff notwendig, ja z.T. führt die Begriffsentwickiung auch über einige Stufen. Aber trotz aller methodischer Maßnahmen der Einführungen und Erprobungen des Begriffs (etwa durch Anwendungen), das zentrale Ziel des Unterrichts ist der fertig ausgearbeitete mathematische Begriff.

Demgegenüber wird in der Wissenschaft der mathematische Begriff und die Begriffsdefinition in umgekehrter Weise verstanden. Hier ist die Begriffsdefinition der Startpunkt des Forschungsprozesses. Begriffsdefinitionen werden als implizite bzw. axiomatische Kennzeichnungen genommen, die inhaltlich offen sind und sich während der Forschungstätigkeit mit immer neuen Bedeutungen und andersartigen Interpretationen füllen. Diese offenheit der Begriffsentwicklung ist in dem Sinne grundsätzlich, daß ein Begriff nie endgültig und vollständig ist; jeder Begriff ist im Prinzip weiterentwickelbar.

Diese grundlegende Verschiedenheit zwischen Wissenschaft und Schule führt im Unterricht oft zu einer folgenschweren Gleichsetzung des mathematischen Begriffs mit seiner (zeichenmäBigen) Darstellung (STEINBRING 1984 und 1985). Die SchuTma- 
thematik hat nicht die Möglichkeit, wie es in der Wissenschaft Praxis ist, die Beziehung zwischen dem Begriff und seiner jeweiligen Definition offenzuhalten. Die Vorgehensweise der Wissenschaft, einen Begriff axiomatisch zu kontrollieren und ihn im ProzeB der Forschungstätigkeit inhaltlich zu entwickeln, ist im allgemeinbildenden Unterricht nicht durchführbar. Begriffe der Schulmathematik beanspruchen in einer relativ konkreten Weise inhaltliche Bedeutung. Anders als in der Wissenschaft muß man im. Mathematikunterricht sagen können, was unter einem bestimmten mathematischen Begriff inhaltlich zu verstehen ist.

Die Frage danach, was ein Begriff ist, muB in Schule und Wissenschaft verschieden beantwortet werden. Die Antwort der Wissenschaft verweist auf die Entwicklungsmöglichkeit des Begriffs, auf seine vielfältigen Bezüge zu anderen Begriffen und auf die neuen Methoden, welche dieser Begriff in den ForschungsprozeB einführt. Eine solche Antwort kann der Unterricht nicht geben. Seine Antwort auf die Frage nach der Begriffsbedeutung ist jedoch oft kurzschiussig: der mathematische Begriff erhält durch seine Definition inhaltliche Bedeutung. Diese Antwort erscheint gerechtfertigt vor dem Hintergrund, daß der schulmathematische Grundbegriff, nämlich der Mengenbegriff, selbstevident ist, und alle anderen Begriffe stellen ja mehr oder weniger Spezifikationen dieses Begriffs dar.

Auch im Unterricht muß man zwischen Begriff und Definition unterscheiden. Bedeutungen schulmathematischer Begriffe ergeben sich nicht einfach aus ihren Definitionen; sie sind auch nicht das Ergebnis individueller Forschungstätigkeiten der einzelnen Schuiler. Im Unterricht gewinnen mathematische Begriffe ihre Bedeutung erst aus einer Vielfalt von intersubjektiven, sozialen und gegenständlichen Tätigkeiten der Schuiler (vgl. Z.B. DORFLER 1984a und 1984b) und durch eine Diversifikation von begrifflichen Darstellungs- und Arbeitsmitteln. Trifft man diese Unterscheidung zwischen Begriff und Definition nicht, so führt dies zu einer Obermethodisierung unterrichtlicher MaBnahmen. Alle Lernaktivitäten sind nicht auf den eigentlichen Begriffsinhalt gerichtet, sie orientieren sich ausschließlich an der speziellen Definitionsform. Daraus ergibt sich die häufig beklagte Künstlichkeit des Mathematikunterrichts, zu der die Mehrheit der Schüler keinen Zugang findet (ANDELFINGER 1985).

Gegenüber diesen Problemen des Mathematikunterrichts ist von seiten der Mathematikdidaktik versucht worden, mit vielfältigen Reformansätzen einer zu starken Strukturorientierung in der SchuTmathematik entgegenzuwirken und sie vermehrt in den sozialen Kontext der Lernsituation einzubeziehen. Dabei zeigt sich, da $\beta$ der augenblickliche Unterricht recht resistent gegen solche Vorschläge ist, sei es projektorientierter und fächerübergreifender Unterricht, sei es problem- oder anwendungsorientierter Unterricht. So stellt KAISER in einer Untersuchung fest, daß Anwendungen zunächst einmal zusätzlichen Stoff bedeuten und - wenn überhaupt - 
nur als gesonderte Unterrichtseinheit durchgeführt werden. Bei vielen Lehrern gibt es grundsätzliche Barrieren gegenüber dem Konzept eines anwendungsorientierten Mathematikunterrichts. "Solche Barrieren sind wohl nicht nur die Stofffülle, der Zeitdruck und das Fehlen geeigneter Beispiele, sondern auch .... die nötige breite Vorbereitung und die Notwendigkeit von Kenntnissen aus anderen Bereichen. ... Des weiteren dürften auch schlechte Erfahrungen, die Lehrer gemacht haben, eine Rolle spielen, nämlich, daß die Schüler eben nicht, wie erhofft, sofort euphorisch reagierten." (KAISER, S.84) Beispielhaft wird klar, auf welche grundsätzlichen Probleme in der etablierten Schulmathematik didaktische Reformansätze stoßen. Nur mit der langsamen Veränderung dieser Grundauffassungen werden sich auch andere Unterrichtsformen beständig durchsetzen können.

Die Mehrzahl der Reformvorschläge zum Mathematikunterricht unterschätzt, daß man nur dann eine wirksame Wechselbeziehung zwischen dem mathematischen Inhalt und den speziellen Bedingungen einer didaktischen Lernsituation herstellen kann, wenn man bewußt eine Verallgemeinerung und Ausweitung von Arbeits- und Darstellungsmitteln sowie von Wissensbedeutungen und Begriffsinterpretationen für die Schulmathematik vornimmt. So fordert z.B. BIEHLER die Entwicklung graphischer Darstellungsmittel als zentrale Erkenntnismittel. "Graphische Darstellungen können und müssen nicht nur als gesellschaftliche Mittel des Lernens und Kommunizierens gesehen werden, sondern auch als Erkenntnismittel der Mathematik als Wissenschaft und dabei vor allem der angewandten Mathematik. Das weitgehende Fehlen dieser Perspektive ist sowohl durch die curriculare Orientierung an der reinen Mathematik, die auch durch die Adjungierung von Anwendungsbeispielen nicht schon aufgehoben ist, als auch durch die medienpädagogische Interpretation von graphischen Darstellungen als methodisches Mittel bedingt. Es kommt jedoch noch ein weiteres hinzu: In der neueren angewandten Mathematik hat sich die Rolle graphischer Darstellungen selbst erheblich verändert ....

Eine neu zu definierende Wissenschaftsorientierung des Mathematikunterrichts müßte auch eine Orientierung an den Mitteln wissenschaftlichen Umgehens mit Mathematik einschließen." (BIEHLER 1985a, S.23/4; vg1. hierzu auch den Aufsatz von FISCHER 1984)

Die Kodifizierung schulmathematischen Wissens und die Auswahl zulässiger mathematischer Arbeits- und Darstellungsmittel darf sich nicht nur an dem augenblicklich festgefügten $B i l d$ von reiner Mathematik orientieren. Mathematische Begriffe und Arbeitsmittel für den allgemeinbildenden Unterricht müssen das breite Spektrum historischer Entwicklungen, philosophischer und wissenschaftstheoretischer oberlegungen, die Spannung zwischen reiner und angewandter Mathematik, sowie neue mathematische Entwicklungen (wie beispielsweise die EDA siehe BIEHLER 1982 und 1985b) beriicksichtigen. Nicht in der Beschränkung auf spezielle Techniken 
der reinen Mathematik, nur durch die Herausarbeitung der mathematischen Erkenntnistätigkeit als einer allgemein gesellschaftlichen Tätigkeit auf der Basis der Vielfalt dieser Aspekte ist es möglich, eine wirksame Wechselbeziehung zwischen der Schuilertätigkeit im Lernprozeß und der mathematischen Erkenntnistätigkeit herzustellen. Erst auf diese Weise wird die Schulmathematik zu einem tatsächlichen und wirkungsvollen Teilsystem im Gesamtsystem der Mathematikdidaktik; nur so kann sich eine echte Wechselbeziehung zu den anderen Teilsystemen herstellen, wenn der Lerngegenstand nicht ein ausschließlich von der Wissenschaft vorgegebenes System mit unveränderlicher Struktur bleibt.

Am Beispiel des Wahrscheinlichkeitsbegriffs soll im folgenden Abschnitt diskutiert werden, wie dieser Begriff seine spezifische Bedeutung erst uiber die Vielfalt von Arbeits- und Darstellungsmitteln erhält, die den Bezug zu den Lerntätigkeiten der Schüler ermöglichen. Des weiteren soll deutlich werden, daß die mathematischen Tätigkeiten für bedeutungsvolles Lernen in der Stochastik immer einen objektiven Gegenstand benötigen, welcher "von außen" der mathematischen Struktur Bedeutung gibt. Die Rolle von Darstellungs- und Tätigkeitsmitteln sowie des Gegenstandes der Mathematik hat häufig in der didaktischen Diskussion zu Begriffslernen vorbereitenden Charakter oder bleibt zumindest implizit (vgl. DORFLER 1984a und 1984b, VOLLRATH 1984, WINTER 1983b).

\section{Wahrscheinlichkeit - Ein neues Paradigma für mathematische Begriffe im Schul-} curriculum?

Für eine vergleichende Analyse sind solche Beispiele von Interesse, die auf Grund ihrer Neuheit und Besonderheit einen erkenntnisgewinnenden Kontrast zu gewohnten Fakten darstellen. Die Wahrscheinlichkeit ist ein solches Beispiel für das traditionelle Schulcurriculum.

Der Wahrscheinlichkeitsbegriff hat eine merkwürdige Eigenschaft: Sobald man versucht, inn mit Hilfe einer endgültigen Definition eindeutig zu charakterisieren, bleibt sofort nichts Stochastisches mehr übrig. Die Zufälligkeit hat ausgespielt. Man hat es nur noch mit sicheren mathematischen Fakten und wohlbestimmten Rechenabläufen zu tun. Was übrigbleibt, sind Mengenlehre, Bruchrechnung bzw. Prozentrechnung, kombinatorische Regeln und Zählverfahren, Aspekte der elementaren Geometrie, einfache Rechenalgorithmen usw.

Die Exaktheitsanforderungen einer mathematischen Definition und der Zufallscharakter von Wahrscheinlichkeit stehen in einem gewissen Widerspruch. Hier wird besonders deutlich $k l a r$, daB die Wahrscheinlichkeit nicht auf ihre mathematische Definition reduzierbar ist. Ein Begriffsverständnis läßt sich nicht einfach auf Grund der Definition geben, so wie dies die Schulmathematik bei ihren mathemati- 
schen Begriffen beansprucht. Schon mit dieser Besonderheit der Stochastik haben Lehrer große Probleme. Sie spüren, daß trotz sorgfältiger Einführung und mathematischer Ausarbeitung der Wahrschein1ichkeit ein Begriffsverständn is bei ihren Schiilern nicht einfach durch die Definition ermöglicht wird. Die Schwierigkeit liegt nicht in der Rekapitulation einer Definition, sondern in ihrer angemessenen Interpretation und situationsspezifischen Anwendung. Dabei ergeben sich aber immer wieder andere und neuartige Deutungen für die Wahrscheinlichkeit. Eine erschöpfende und definitive Begriffsklärung, auf die der Unterricht üblicherweise abzielt, ist beim Wahrscheinlichkeitsbegriff prinzipiell nicht möglich. Die Frage: "Was ist Wahrscheinlichkeit?" kann nicht nach dem gleichen Muster wie für die anderen Begriffe der Schulmathematik beantwortet werden.

Diese Differenz zwischen ihrer mathematischen Charakterisierung und der besonderen stochastischen Bedeutung von Wahrscheinlichkejt ist der Kern für viele didaktische Schwierigkeiten mit diesem ungewöhnlichen Schulstoff. Wir wollen dies als Ausgangspunkt nehmen, um eine neue Sichtweise auf mathematische Begriffe im Schulcurriculum zu gewinnen. Unser Ziel ist nicht nur ein Plädoyer für den Stochastikunterricht. Durch die kontrastierende Gegenüberstellung der Wahrschein1ichkeit mit herkömmlichen mathematischen Begriffen, welche sich auf fachliche, historische, wissenschaftstheoretische sowie didaktische und unterrichtspraktische Oberlegungen bezieht, soll beispielhaft die Vielfalt der mathematischen Erkenntnistätigkeit analysiert und der mathematische Begriff als ein beziehungsreiches System mode1liert werden.

\subsection{Begriff und Bedeutungsvielfalt}

Die Frage nach der Bedeutung des Wahrscheinlichkeitsbegriffs hat in der historischen Entwicklung heftige Kontroversen ausgelöst, hat in der Philosophie zu fundamentalen Auseinandersetzungen geführt, hat die Grundlagendiskussion der $\mathrm{Ma}-$ thematik um die Jahrhundertwende beeinflußt, hat wissenschaftstheoretische Erörterungen über den Status wissenschaftlicher Begriffe ausgelöst und hat auch in der didaktischen Diskussion gegensätzliche Standpunkte hervorgebracht. Die Antworten auf diese Fragen sind vieldeutig, widersprüchlich und von völlig unterschiedlichem Status. Allseits bekannt sind beispielsweise folgende gegensätzliche Außerungen:

- Wahrscheinlichkeit ist ein Konstrukt des Erkenntnissubjekts, welches dieses Konzept in der Auseinandersetzung mit der Umwelt schafft. Demgegenüber: Wahrscheinlichkeit ist eine Eigenschaft bestimmter objektiver Mechanismen, die wie Zufallsgeräte funktionieren.

- Wahrscheinlichkeit ist ein Begriff der beobachtbaren Realität; er wird als 
Grenzwert von relativen Häufigkeiten interpretiert. Demgegenüber: Wahrscheinlichkeit ist ein idealer Begriff; er wird durch bestimmte ideale Symmetrien konstituiert.

- Wahrscheinlichkeit ist ein Hilfsbegriff, der bei unvollständigem Wissen des Erkenntnissubjekts zu Rate gezogen wird. Hat man über die betrachtete situation ausreichendes Wissen, dann ist die Wahrscheinlichkeit uberflüssig. Demgegenüber: Alles Wissen über die Wirklichkeit ist letztlich mit Unsicherheiten behaftet; daher ist der Wahrscheinlichkeitsbegriff der fundamentalste Begriff wissenschaftlicher Erkenntnis.

- Der Wahrscheinlichkeitsbegriff ist nur in speziellen Massenexperimenten aussagefähig; auf den Einzelfall ist er nicht anwendbar. Demgegenüber: Wahrscheinlichkeit ist ein Begriff des Alltagswissens, unmittelbar nuitzlich und für alle Situationen verwendbar.

- Wahrscheinlichkeit ist ein theoretischer Begriff, seine Bedeutung läßt sich nicht direkt erschließen, sie ergibt sich erst in der Konstruktion und Anwendung stochastischer Modelle. Demgegenüber: Wahrscheinlichkeit ist ein empirischer Begriff, seine Bedeutung ergibt sich in Durchführung und Auswertung eines statistischen Experiments.

Gegensätzliche Standpunkte dieser Art haben zu einer mannigfaltigen Aufsplitterung der Wahrscheinlichkeit in eine schillernde Vielfalt eigenständiger Begriffe geführt. Es finden sich Typen von axiomatischer Wahrscheinlichkeit, von komparativer Wahrscheinlichkeit, Häufigkeitsinterpretationen der Wahrscheinlichkeit, klassische (Laplacesche) Wahrscheinlichkeiten, die logische Wahrscheinlichkeit, informationstheoretische Zugänge zur Wahrscheinlichkeit, komplexitätstheoretische Begründungen der Wahrscheinlichkeit, Wahrscheinlichkeit als subjektive Evidenzen usw. usf. (vgl. hierzu z.B. FINE 1973).

Jede dieser Varianten gibt der Wahrscheinlichkeit eine spezifische Bedeutung und interpretiert sie entsprechend eigenen Anforderungen. Eine einheitliche Begriffsauffassung ist angesichts dieses Pluralismus unmöglich.

Betrachtet man demgegenüber den Aufbau des jeweils benötigten mathematischen Kalkïls, so lassen sich Ahnlichkeiten und obereinstimmungen feststellen. Der mathematische Apparat aller Wahrschein1ichkeitsbegriffe ist vergleichbar und vor einer gemeinsamen Struktur - 2.B. der Axiomatik Kolmogoroffs - aufeinander beziehbar.

Diese Tatsache mag für einige Wahrscheinlichkeitstheoretiker AnlaB gewesen sein, überhaupt nicht mehr nach auBermathematischen Bedeutungen der Wahrscheinlichkeit zu fragen, sondern einfach auf die Axiomatik zu verweisen bzw. die Grundregein der Wahrscheinlichkeitsrechnung zu nehmen. In der Stochastikdidaktik war man an- 
fänglich bemüht, Z.B. durch die Boolesche Algebra interne fachliche Begründungsstrukturen für den Wahrscheinlichkeitsbegriff aufzubauen (vgl. z.B. WINKELMANN, S.267). Die Bedeutungsvielfalt der Wahrscheinlichkeit sollte eingeschränkt und durch formalisierte Beziehungen erklärt werden.

Welche Auswirkungen hat die Bedeutungsvielfalt der Wahrscheinl ichkeit für das mathematische Curriculum? Der allgemeinbildende Mathematikunterricht darf nicht eine einzige Deutung des Wahrscheinlichkeitsbegriffs zur Grundlage machen. "Stochastik auf der Schule muß Vorstellungen aus verschiedenen philosophischen Traditionen organisch miteinander verbinden, insbesondere

- Stochastik als die Mathematik der Massenerscheinungen

- Stochastik als die Logik der Ungewißheit

- Stochastik als die Technologie, die Daten in Erkenntnisse ummünzt

- Stochastik als Entscheidungstheorie."

(DINGES $1981, \mathrm{~S} .51$ )

Auch für den Mathematikunterricht muß man aus der Begriffsvielfalt die Konsequenz ziehen, daB es keine universelle und einzigartige Interpretation der Wahrscheinlichkeit gibt. Dieser Begriff besteht einerseits aus einer mathematischen Struktur (z.B. einer Axiomatik oder impliziten, definierenden Gleichungen oder Regeln des Kalkiils) und andererseits bezieht er sich auf verschiedenartigste und spezifische inhaltliche Deutungen (vgl. v.HARTEN/STEINBRING, S.64/5, JAGER/SCHUPP 1983).

$\mathrm{Da}$ die mathematische Begriffsstruktur verschiedene inhaltliche Interpretationen zuläßt, kann die Bedeutung des Wahrscheinlichkeitsbegriffs nicht ausschließlich Ergebnis innermathematischer Oberlegungen sein. Für den Wahrscheinlichkeitsbegriff wird seine Interpretation zu einem eigenständigen Problem, das zusätzlich zur Mathematik hinzutritt.

Die Bedeutung des Wahrscheinlichkeitsbegriffs erschöpft sich weder allein in der mathematischen Struktur - was ihn im Schulcurriculum beispielsweise auf Boolesche Algebra oder Kombinatorik reduzieren würde -; noch erhält dieser Begriff durch unmittelbare empirische Erfahrungen und Experimente seine Bedeutung -, die die Stochastik schnell zu einer Rezeptsammlung einfacher Rechentechniken werden 1äßt. Im Unterschied zu den traditionellen Begriffen des Schulcurriculums benötigt der Wahrscheinlichkeitsbegriff neben seiner mathematischen Definition explizit einen Referenzrahmen für inhaltliche Interpretationen und Anwendungen. Die Bedeutung der Wahrscheinlichkeit wird weder durch die sachlogische Struktur noch durch die Anwendungssituationen automatisch mitgeliefert.

Auf diese Besonderheit der Stochastik haben auch bekannte Wahrscheinlichkeits- 
theoretiker hingewiesen. So stellt Kolmogoroff in seiner Grundlegung der Wahrscheinlichkeit neben die Formulierung der Axiome dieses Begriffs seine Oberlegungen zum Thema "Das Verhältnis zur Erfahrungswelt". Hier wird eine spezifische anwendungsorientierte Deutung der Wahrscheinlichkeit diskutiert, die sich an der Häufigkeits interpretation orientiert (KOLMOGOROFF, S.3f). In einer ähnlichen Weise betont FELLER in der Einleitung zu seinem Lehrbuch, daß zusätzlich zur mathematischen Definition von Wahrscheinlichkeit Anweisungen über inhaltliches Verständnis und Gebrauch dieses Begriffs notwendig sind. FELLER sagt: "In a rough way we may characterize this concept by saying that our probabilities do ... refer ... to possible outcomes of a conceptual experiment. Before we speak of probabilities, we must agree on an idealized model of a particular conceptual experiment such as tossing a coin, sampling kangaroos on the moon, observing a particle under diffusion, counting the number of telephone calls." (FELLER, S.4)

Die dargestellte Begriffsvielfalt der Wahrscheinlichkeit in wissenschaftlichen Diskussionen kann man auch im Schulunterricht wiederfinden. So werden vor allem jüngere Schüler, die sich noch nicht an die Standards schulmathematischer Argumentationen gewöhnt haben, auf die Fragen nach der Begründung von Wahrschein1 ichkeiten in konkreten Zufallsexperimenten sich vielfältiger inhaltlicher Bedeutungserklärungen bedienen. Auf die Frage: Ist es wahrscheinlicher, mit einem (idealen) Würfel eine 4 oder eine 6 zu würfeln? gaben beispielsweise Schüler einer 6. Klasse Antworten, wie sie in anderer Form auch in wissenschaftstheoretischen Diskussionen auftauchen. Einige Schüler meinten, das kann man nicht sagen, da dies vom Zufall abhängt, andere Schüler sahen von den angenommenen Symmetriebedingungen des Würfe $7 s$ her keine Unterschiede für die 4 und die 6 , während ein Schüler vorschlug, man solle erst einmal tausendmal werfen, um dann nachzusehen, ob 4 und 6 gleichhäufig auftreten. Die Schüler, so könnte man sagen, wählten jeweils bestimmte Interpretationsperspektiven für die Wahrschein1ichkeit: Sie orientierten sich an den physikalischen Bedingungen des Zufallsgerätes, sie interpretierten Wahrscheinlichkeit im Sinne eines wiederholbaren Massenexperiments, oder sie deuteten Wahrscheinlichkeit als eine rein subjektive und von augenblicklichen Zufällen abhängige Größe.

Die Tatsache, daB zur mathematischen Definition von Wahrscheinlichkeit immer auch heuristische Vorstellungen und inhaltiiche Interpretationen gehören, macht die Stochastik zu einem besonderen Typ anwendungsorientierter Mathematik. Anwendungen der mathematischen Stochastik können nicht einfach im Sinne klassischer mathematischer Anwendungen aufgefaßt werden, die erst nach der Entwicklung einer ausgebauten Theorie einsetzen. Umgekehrt, hier ist der Anwendungsbezug Voraussetzung für die Entwicklung des mathematischen Begriffs. Bezogen 
auf das Verhältnis von Begründung und Anwendung muß man im Stochastikunterricht bewußt eine "historische Perspektive", einen Entwicklungsstandpunkt einnehmen, andernfalls verliert der Wahrscheinlichkeitsbegriff seinen besonderen Charakter. Eine solche Vorgehensweise wäre auch für andere Begriffe des Schulcurriculums möglich und wichtig; diese werden jedoch häufig "strukturell" erarbeitet und weniger im Kontext von Anwendungsbezijgen entwickelt.

Ein solcher Typ pragmatischer und unmittelbarer Anwendungen mathematischer Begriffe bringt eine Verschiebung des Bedeutungsproblems für den Mathematikunterricht mit sich. Bedeutungen der Wahrscheinlichkeit ergeben sich nicht auf Grund ausgearbeiteter theoretischer Zusammenhänge; sie können auch nicht das Ergebnis einer Vereinheitlichung der vielfältigen inhaltlichen Interpretationen von Wahrscheinlichkeit sein (vgl. auch DINGES 1981, SCHUPP 1982).

Die spezifische Bedeutung der Stochastik ergibt sich aus dem System von Tätigkeiten und Darstellungsmitteln, die stochastisches Arbeiten in diesem experimentell anwendungsorientierten Sinne ermöglichen. In dem Spektrum spezifischer mathematischer und allgemeiner erkenntnisorientierter Darstellungsmittel und Tätigkeiten läßt sich für den Mathematikunterricht die besondere Bedeutung des Wahrscheinlichkeitsbegriffs charakterisieren ( $v g l$. hierzu auch die Deutung des Wahrscheinlichkeitsbegriffs als "Handlungssystem" bei DORFLER 1985, S.7ff). Begriffsbedeutungen sind für den Schüler nicht Ergebnis einer Vereinheitlichung der Begriffsdefinition, sondern nur durch die Ausweitung der zum Begriff gehörenden Darstellungs- und Tätigkeitsmittel möglich.

\subsection{Begriff und Mittel}

Stochastisches Denken kann man als Inbegriff des theoretischen Denkens überhaupt auffassen. Wie ist diese Aussage zu verstehen? Im Vergleich mit anderen Denkweisen, z.B. der geometrischen, algebraischen oder mechanischen, ist man bei stochastischem Denken von vornherein gezwungen, bewußt den Unterschied zwischen mathematischem Modell und realer Situation zu beachten. Diese "distanzierte Rationalität" (DINGES 1982) zwischen mathematischer Struktur und Wirklichkeit stellt sich in der stochastischen Erkenntnis quasi von selbst ein. Stochastische Aussagen über reale Phänomene sind keine Abbilder empirischer Wahrheiten, sie reflektieren vor allem mögliche theoretische Zusammenhänge in der vorliegenden Situation. Ausdruck ist hierfür die Tatsache, daß stochastische Aussagen über die Wirklichkeit immer mit einer sogenannten Irrtumswahrschein1ichkeit behaftet sind (DINGES 1981, STEINBRING 1980b). Egal wie man dies im Hinblick auf den epistemologischen Status der Wahrscheinlichkeit interpretiert - ob man hierin den Hilfscharakter oder den konzeptionellen Fortschritt der Stochastik erblickt - in jedem Falle verhütet dies die direkte Identifikation 
der mathematischen Aussage mit dem realen Sachverhalt, die Gleichsetzung von Zeichen und Gegenstand.

Diese Besonderheit ist nicht nur ein Problem in wissenschaftstheoretischen Diskussionen um das Verhältnis von praktischer Statistik zur Wahrscheinlichkeitstheorie oder bei umfassenden Anwendungen der Stochastik, sie tritt auch im Mathematikunterricht in Form der Beziehung von konkretem Einzelfall zum stochastischen Modell zutage (vgl. auch SCHUPP 1982).

Ein einfaches, aber aussagekräftiges Beispiel im Mathematikunterricht ist die Anfertigung eines "idealen" Glücksrades. Zunächst gehen Modellvorstellungen über mechanische und symmetrische Bedingungen in die Konstruktion ein: Aufteilungen der Kreisscheibe (z.B. ein Bierdeckel) in gleichgroBe Sektoren, Anbringen der Drehachse im Mittelpunkt der Scheibe, usw. Wenn das Glücksrad fertiggestellt ist, müssen die Schüler im zweiten Schritt dieses Gerät als einen konkreten Einzelfall untersuchen. Im Verlaufe experimenteller Erprobungen lassen sich Abweichungen von der sog. idealen Symmetrie feststellen und daraus evtl. Rückschluisse auf Konstruktionsfehler ziehen.

So erkannten beispielsweise Schüler im Unterricht, daß die experimentell beobachteten Unterschiede in den relativen Häufigkeiten bei ihren Geräten von der ungenauen Bestimmung des Mittelpunktes herruhrten. Eine Korrektur führte zu Experimentergebnissen, die eher der Verteilung eines "idealen" Gluicksrades entsprachen. Zudem änderten diese Schüler auch die "Durchführungsanweisungen" für das Experiment: Die rotierende Scheibe sollte nicht mehr senkrecht gehalten, sie sollte nur noch in wagerechter Position gedreht werden. Erprobungen am konkreten Einzelfall und allgemeine ideale Vorstellungen zum Modellfall führten so wechselweise zu einer Verbesserung des Zufallsgerätes und zu ersten elementaren Einsichten in die Beziehung mechanischer Faktoren und zufallsbedingter Parameter.

In diesem, wie in vielen weiteren Beispielen, läßt sich der wechselseitige ErkenntnisprozeB jedoch nicht automatisieren. Es bleibt letztlich immer eine bewuBte Entscheidung des Subjekts, ob es beobachtete Abweichungen als relevant oder als zufällig und damit vernachlässigbar interpretieren will - ob dies nun vor dem Hintergrund umfangreicher statistischer Techniken geschieht oder ob es sich um elementare stochastische Zusammenhänge im Mathematikunterricht handelt. Ein weiteres Beispiel hierfür ist die Frage, wann man einen Würfel als gefälscht oder ideal ansehen will, bzw. welche Wahrscheinlichkeitsverteilung man einem gefälschten Würfel zuordnen möchte. Auch dies ist nicht einfach eine Frage an die statistischen Methoden; darüber hinaus spielen mechanische Parameter wie die Schwerpunktlage des Würfels eine wichtige Rolle. So äußern bei- 
spielsweise Schüler durchaus angemessene Vermutungen über die zu erwartenden Fälschungen eines Würfels auf Grund mechanischer Defekte. Interessant ist es auch, Schüler aufzufordern, einen in bestimmtem Umfang gefälschten Würfel selbst zu bauen.

Vor allem jüngere Schüler sind meist bereit, in stochastischen Situationen die Beziehung von Modell und Einzelfall in beispielhafter Form für ihre Argumentation aufzugreifen. Sie verlassen sich etwa nicht nur auf allgemeine mathematische Begründungen, sondern berücksichtigen auch spezifische Aspekte der konkreten Sachlage. Zugleich ist es manchmal verwunderlich, an welchen Stellen sich Schuiler über konkrete Einzelfälle eine "Theorie" zu machen versuchen. Bekanntlich wird z.B. das Reißnagelexperiment als das statistische Experiment an sich angesehen, das keine ideale Modellvorstellung gestattet. Ein Schüler stellte demgegenüber jedoch eine plausible Modellannahme auf, die durchaus stochastische Prognosen zuließ. Er schlug vor, Reißnägel mit langen Dornen, etwas kürzeren, normal langen, sehr kurzen Dornen und ohne Dornen zu betrachten. Diese model 1hafte Variation des Parameters "Länge des Reißnageldorns" ermöglichte komparative stochastische Aussagen, die nicht ausschließlich auf experimentellen Ergebnissen beruhten.

Diese Beispiele belegen anschaulich, welche Grundbeziehung in der Analyse stochastischer Zusammenhänge zentral ist: die Wechselbeziehung zwischen stochastischem Modell und konkretem Einzelfa11. Weder ergibt sich das Modell automatisch aus dem Experiment des Einzelfalls, noch ist das Modell eine vollständige Erklärung aller konkreten Phänomene des Einzelfalls. Für den Mathematikunterricht ist es ein völlig ungewohntes Faktum, daß sich die empirischen Einzelheiten nicht auf eine allgemeine mathematische Struktur reduzieren lassen. Die Stochastik bringt auf diese Weise eine strikte Trennung von Zeichen und Bezeichnetem oder von mathematischer modellmäßiger Beschreibung und Gegenstand in den Unterricht. Demgegenüber gibt es in den traditionellen Stoffgebieten des Schulcurriculums häufig eine Identifizierung von mathematischem Zeichen und Gegenstand.

Geht man davon aus, daß sich die Beziehung von Einzelfall zu Modell nicht reduktionistisch auflösen läßt, so stellt sich, bezogen auf den Unterricht, die Frage nach den Bedingungen der mathematischen Bearbeitung und Handhabung dieser stochastischen Beziehung. In der Geschichte der Wahrscheinlichkeitstheorie ist eine Vielfalt an konzeptionellen Beschreibungs- und Darstellungsmitteln entwickelt worden, die insbesondere den besonderen Typ zwischen System und Systemelement charakterisieren. An erster Stelle ist hier der Begriff der Verteilung zu nennen ( $v g l$. SAČKOV 1978). 
Bei genauerer Betrachtung lassen sich zwei Formen von Darstellungsmitteln zur Analyse der stochastischen Beziehung zwischen Einzelfall und Modell erkennen. Zur Sorte der Verteilung gehören alle die Diagramme, welche es primär gestatten, Ergebnisse von Häufigkeitsexperimenten aufzubereiten. Im Unterricht hat man hier: Tabellen, Strichlisten, Kreisdiagramme, Stengel- und Blätter-Schaubilder, Stabdiagramme, Säulendiagramme, Histogramme, Polygonzüge usw.

Zum anderen gibt es Diagramme, die sich vornehmlich auf den Kalkül der Wahrscheinlichkeit beziehen; typisch hierfür ist das sog. Baumdiagramm. Hierzu lassen sich im Unterricht folgende Formen zählen: Tabellen (Matrizen), vollständiges Baumdiagramm, reduziertes Baumdiagramm, "unendliches" Baumdiagramm, "rekursives" Baumdiagramm, Pascalsches Dreieck, Wahrscheinlichkeitsabakus usw.

Diese beiden Typen stochastischer Darstellungsmittel werden im Unterricht meist strikt ihren Intentionen gemäß entweder als Hilfsmittel zur Aufbereitung von Daten oder als Hinführung zur mathematischen Rechenformel eingesetzt. Bezogen auf die Beziehung von Einzelfall zu Modell ist es jedoch angebracht, Verteilung und Baumdiagram als komplementäre Darstellungsmittel aufzufassen. Das heiBt beispielsweise, den Verteilungsbegriff sollte man auch für die Berechnung und Darstellung von Wahrscheinl ichkeiten nutzen und umgekehrt, Darstellungsmittel aus der Kategorie Baumdiagramm, wie z.B. das Pascalsche Dreieck, sollte man zur Erklärung für bestimmte Verteilungen heranziehen.

Kurz, es gilt ein Zusammenwirken aller stochastischer Darstellungsmittel zu entwickeln, das es gestattet, wechselweise den Obergang vom Einzelfall zum Modell zu vollziehen. Jedes der aufgeführten Darstellungsmittel thematisiert in spezifischer Weise die Beziehung von Einzelfall zu Model1. So läßt sich das Baumdiagramm gleichzeitig als Ablaufplan für die Durchführung eines konkreten Experiments und als eine model Thafte Charakterisierung aller auftretenden Möglichkeiten interpretieren. Der Verteilungsbegriff ist 2.B. eine Darstellungsform, die das Gesamtsystem aller Zufallsgrößen charakterisiert und gleichzeitig anhand einzelner ausgezeichneter Werte der Verteilung (z.B. der Symmetrie, der Mittelwerte oder Streuwerte usw.) allgemeine theoretische Aussagen uber einen Einzelfall macht. Diese Doppelrolle gibt allen stochastischen Darstellungsmitteln einen gemeinsamen Bezugspunkt. Im Unterricht mußten sie aufeinander abgestimmt und didaktisch organisiert werden. Erst das System der stochastischen Darstellungsmittel ermöglicht eine angemessene stochastische Deutung der Beziehung zwischen Modell und Einzelfall.

Im Rahmen einer didaktischen Organisation der stochastischen Darstellungsmittel zueinander sind beispielsweise Verwendungsformen auf unterschiedlichen Ebenen ratsam: die Benutzung der Diagramme zur Beschreibung, Durchführung und Auswer- 
tung realer Zufallsexperimente, zur Simulation von Experimenten, zur Durchführung und Auswertung idealer Massenexperimente, zur Darstellung rekursiver Schemata (z.B. Binomialkoeffizienten), zur Herleitung einfacher FormeIn der Stochastik (z.B. Pfadregeln), zur Aufstellung eines stochastischen Modells (z.B. Binomialverteilung) usw.

Eine so verstandene systematische Variation der stochastischen Darstellungsmittel garantiert, daB die Spannung zwischen konkreter Zufallssituation und ihrer stochastischen Modellierung aufrechterhalten bleibt. Das System der Darstellungsmittel wird so zum Träger der spezifischen stochastischen Bedeutung, die in der jeweiligen Anwendungssituation eine Rolle spielt, und es beinhaltet die Möglichkeit einer gleichzeitig model Thaften wie konkreten stochastischen Interpretation dieser Situation.

Die für das stochastische Denken fundamentale Beziehung von konkretem Einzelfall zu stochastischem Modell führt in den Unterricht in grundsätzlicher Weise die Trennung zwischen mathematischem Zeichen und bezeichnetem Gegenstand ein. Zur angemessenen mathematischen Handhabung der Beziehung zwischen Einzelfall und Modell ist das System stochastischer Darstellungsmittel (Baumdiagramme und Verteilungen) notwendig. Dem System dieser Darstellungsmittel korrespondiert eine Vielfalt an Gebrauchsweisen durch das Erkenntnissubjekt; durch den varijerenden Gebrauch der Diagramme wird das System der Darstellungsmittel zum Träger der spezifischen stochastischen Bedeutung ( $v$ gl. BIEHLER 1985a, FISCHER 1984). In der Verwendung spezifischer stochastischer Darstellungsmittel zur Oberbrïkkung des Verhältnisses von Einzelfall und Modell konstituiert sich Bedeutung und Begrifflichkeit der Stochastik. Man gelangt erst über die Trennung von Gegenstand und Zeichen zum mathematischen Begriff. Für diese dreifache Unterscheidung zwischen Begriff, Zeichen und Gegenstand soll im folgenden Abschnitt ihre wechselseitige Bezugsstruktur im didaktischen Lernprozeß analysiert werden.

\subsection{Begriff und Lernen}

Der mathematische Begriff ist nicht mit seiner Definition identisch; zudem unterscheidet er sich vom Lern-Gegenstand. Wodurch konstituiert sich dann der Begriff im LernprozeB, wenn er weder seiner Definition (den mathematischen Zeichen) noch dem Gegenstand gleich ist?

Der Begriff entsteht in der aktiven Auseinandersetzung des Subjekts mit der Beziehung zwischen Gegenstand und mathematischen Zeichen, in der wechselseitigen Anwendung von jeweiliger mathematischer Definition und konkretem Anwendungsfall. Für den Wahrscheinlichkeitsbegriff vermitteln die stochastischen Darstellungsmittel zwischen konkreter Zufallssituation und der mathematischen Modellierung. 
In diesem Kontext wird die duale Kennzeichnung der Wahrscheinlichkeit für die Aneignung dieses Begriffs nützlich (vgl. hierzu auch SCHUPP 1982). Einerseits hat man eine experimentelle Kennzeichnung der Wahrscheinlichkeit als einem Schätzwert auf Grund beobachteter relativer Häufigkeiten. Zum anderen gibt es eine theoretische Deutung der Wahrscheinlichkeit. Auf Grund inhaltlicher Hypothesen (über Symmetrien, Flächenanteile usw.) wird die Wahrscheinlichkeit durch einen relativen Anteil bestimmt. Der Wahrscheinlichkeitsbegriff beinhaltet so im Keim die duale Struktur mathematisch-modellierender und konkret-experimenteller Aspekte. Diese Begriffsstruktur "entspricht" der Beziehung von Einzelfall und Mode11.

Hieran wird deutlich, wie schädlich es wäre, den Wahrscheinlichkeitsbegriff im Unterricht durch eine einzige Definition ein für allemal festlegen zu wollen. Der Stochastikunterricht sollte sich vielmehr an dem folgenden didaktischen Prinzip orientieren:

Für eine inhaltlich bedeutungsvolle Behandlung der Stochastik im Mathematikunterricht ist es notwendig, den Wahrscheinlichkeitsbegriff gleichzeitig auf einer experimentell-anwendungsbezogenen und einer mathematischen Modellebene zu entwickeln. Jede vorschnelle Entscheidung zugunsten einer einzigen der beiden Kennzeichnungen von Wahrscheinlichkeit und gegen die andere - ob als relative Häufigkeit oder als relativer Anteil - beinhaltet die Gefahr einer Reduktion der Wahrscheinlichkeitstheorie auf formale mathematische Techniken.

Dieses Prinzip steht in gewissem Gegensatz zum verbreiteten Selbstverständnis der Schulmathematik, wonach der mathematische AbstraktionsprozeB von konkreten Beispielen schrittweise zu den eigentlichen Sachverhalten, nämlich Formeln und Begriffen, vorstößt, indem man von situativen Eigenschaften absieht, um dann in der Obungsphase im nachhinein die erlernten Begriffe auf Beispielsfälle anzuwenden.

Stochastikunterricht (in der Sek.I) kann nicht auf einem universellen Prinzip beruhen; er bedarf vielfältiger Zugangs- und unterschiedlicher Arbeitsweisen. Mit seinem Vorschlag für einen "sinnvollen Stochastikunterricht in der Sekundarstufe I" hat SCHUPP (1984) versucht, in Form von sechs Arbeitsphasen die Vielfalt der stochastischen Tätigkeiten in einer Sequenz für den Unterricht zu ordnen. SCHUPP bringt hiermit gleichzeitig zum Ausdruck, daB diese Phasen einander nicht einfach ablösen, sondern jeweils eine neue (stochastische) Aktivität mit bereits bekannten verbinden. So kann man z.B. feststellen, daß die Tätigkeit der ersten Phase: Experimentieren, explizit in allen anderen Phasen benötigt wird. Diese Sequenz stellt keine (strukturelle) Hierarchie dar, sondern betont eher das Moment der Gleichzeitigkeit (in jeweiligem Umfang) von Tätig- 
keiten aus allen sechs Phasen.

Entsprechend dem System stochastischer Darstellungsmittel entsteht im Lernprozeß das Tätigkeitssystem bzw. Handlungssystem (DORFLER 1985, S.8) der Schuiler. In der Auseinandersetzung mit den Darstellungsmitteln und durch ihre spezifische Verwendung führen die Schüler Tätigkeiten auf verschiedenen Ebenen durch: Sie experimentieren, sammeln Daten, vergleichen, führen Auswertungen durch, schätzen, messen, entscheiden, stellen Annahmen und Modelle auf, rechnen mit Formeln, geben mathematische Argumente usw.

Diese Tätigkeiten bedürfen unterschiedlicher Mittel, allgemeiner Mittel der Erkenntnis und auch stochastischer Mittel der Darstellung. Sie durfen nicht hierarchisch strukturiert werden in dem Sinne, daß man eine Einteilung in vorläufige und nebensächliche sowie in richtige mathematische Tätigkeiten vornimmt. Das gesamte Tätigkeitssystem ermöglicht es dem Schüler erst, die stochastische Bedeutung zu erfassen. Die in dem System angelegte Breite stellt eine Verbindung her zwischen dem Alltagswissen der Schüler und dem (spezifischen) mathematischen Wissen.

Am Beispiel des Galtonbrettes sollen diese Oberlegungen konkretisiert werden. Dieser stochastische Gegenstand bietet vielfältige Einstiegsmöglichkeiten und sollte den Unterricht (der Sek.I) beständig begleiten ( $v g$ l. auch JAGER/SCHUPP 1983). Die Doppelrolle, gleichzeitig stochastisches Modell und spezieller Einzelfall zu sein, wird am Galtonbrett prägnant sichtbar. So ergeben sich bei mehreren Versuchen mit diesem Zufallsgerät im Detail immer verschiedenartige Verteilungskurven, die jedoch zugleich gewisse Gleichheiten, allgemeine Ahnlichkeiten bzw. Symmetrien aufweisen. In den Spezialfällen erkennt man einen Modelltyp.

Den Schülern bietet diese Ausgangssituation viele Anlässe, ihr Alltagswissen einzubeziehen, einfache physikalische Argumente vorzutragen und sich gegenstandsbezogene Annahmen zu überlegen. So wird z.B. von Schülern häufig vermutet, die Schwerkraft bewirke die signifikante Häufung der Kugeln in der Mitte der Verteilung. Werden Begründungen nicht ausschließlich auf der Ebene des stochastischen Modells gesucht, sondern der reale Gegenstand auch selbst ernstgenommen, so ist dieses Argument nicht so ohne weiteres von der Hand zu weisen. So verursacht bei einigen Galtonbrettern ein Kippen des Bretts nach hinten eine stärkere Roll reibung der Kugeln; die Schwerkraft wird stärker wirksam: Die Häufung in der Mitte wird noch größer sein als im Normalzustand, bei dem man die Schwerkraft als statistisch vernachlässigbar betrachtet. Bei anderen Galtonbrettern zeigen sich beim Kippen nach hinten genau entgegengesetzte Auswirkungen: Die Anzahl der Kugeln in den Verteilungsschwänzen ist gröBer, die in der Verteilungsmitte geringer als erwartet. Hier muB man davon ausgehen, daB 
auf der "schiefen Ebene" des Galtonbretts die rollenden Kugeln gewisse Stabilitäten in der einmal eingeschlagenen Bewegungsrichtung haben und damit die "geraden" Wege im Galtonbrett gegenüber den "Zick-Zack"-Wegen leicht bevorzugt sind ( $v g 1$. hierzu STEINBRING 1985a).

Stochastische Experimente mit Galtonbrettern unter verschiedenen Versuchsbedingungen zeigen, daß man den Untersuchungsgegenstand ernstnehmen muß, zwar mit Modellvorstellungen an ihn herangeht, ihn jedoch auch selbst "sprechen" lassen muß. Dieses Wechselspiel zwischen Gegenstand und Modell gilt es zu entwickeln, um so stochastische Bedeutungen aufzubauen.

In einer möglichen ersten Arbeitsphase führen die Schüler Experimente mit dem Galtonbrett durch, sameln und ordnen die gewonnenen Daten, um so vergleichende Bewertungen und erste Annahmen für ein mathematisches Modell zu erhalten. Fragen nach inhaltlichen Ursachen für bestimmte sichtbare Phänomene (wie etwa die Symmetrie, die Häufung in der Mitte usw.) lassen sich durch geschickte Variationen des Experiments besser beantworten.

Eine Variation des Experimentes mit dem Galtonbrett ist das Kippen des Brettes nach hinten; in einer anderen Variation kann man zu verschiedenen seitlichen Kippwinkeln (zwischen Auflage und Unterkante des Bretts) Experimente durchführen. Der auswertende Vergleich dieser Experimente erfordert Tätigkeiten des Sammelns, des Ordnens sowie der Darstellung mit Hilfe von Verteilungen. Im AnschluB hieran lassen sich Hypothesen über bestimmte Experimente formulieren:

- z.B. daB man im "Normalzustand" des Bretts eine symmetrische Verteilung vorfindet,

- daß hierbei die Kugeln an einzelnen Zapfen mit gleicher Wahrscheinlichkeit links oder rechts herabfallen,

- daB man beim seitlich gekippten Galtonbrett eine unsymmetrische und seitiich verschobene Verteilung hat (abhängig von der Größe des Winkels),

- daß hierbei die Kugeln an den einzelnen Zapfen nicht mit gleicher Wahrscheinlichkeit links und rechts herabfallen (abhängig von Kippstelle und Kippwinkel fällt die Kugel mit größerer Wahrscheinlichkeit an einer der beiden Seiten des Zapfens herab).

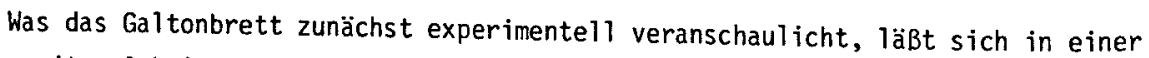
zweiten Arbeitsphase durch eine ideale Simulation beschreiben. Als wichtiges Mittel für die Simulationstätigkeit wird das Pascalsche Dreieck benutzt. Entsprechend den experimentell vermuteten Wahrscheinlichkeiten wird eine große Anzahl (gedachter Versuche) durch das Diagramm "geschoben". Für den Normalzustand werden z.B. jeweils die Hälfte der Anzahlen nach links bzw. rechts weitergeleitet. 


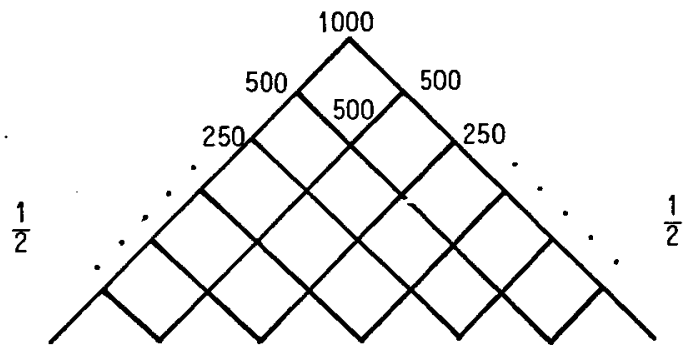

Die ideale Simulation ist ein Bindeglied zwischen realem Experiment, für das das Pascalsche Dreieck hier ein Verlaufsdiagramm darstellt, und einer mathematisch modellmäßigen Berechnung.

In einer dritten Phase lassen sich einfache mathematische Tätigkeiten und Routinen, bezogen auf eine neue Interpretation des Pascalschen Dreiecks, in den Lernprozeß einführen. Der erste wichtige Begriff in diesem Zusamenhang ist die "Anzahl der Wege zu einem Endpunkt". Im Rahmen des numerischen Diagramms "Pascalsches Zahlendreieck" läßt sich dieser Begriff in einer ersten einfachen Form begründen, und es lassen sich spezielle Werte rekursiv ermitteln. Im Vergleich mit der idealen Simulation kann die Besonderheit dieser "stochastischen Tätigkeit" diskutiert werden.

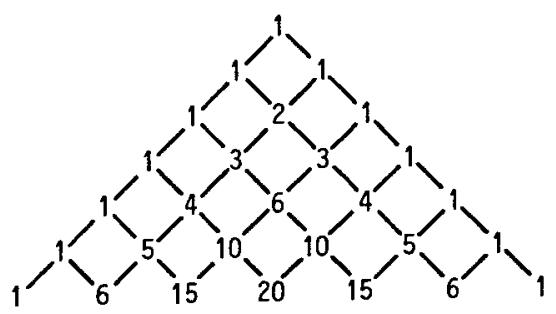

Der zweite wichtige Begriff bezieht sich auf die Pfadregeln (Produkt- und Summenrege1). Diese Regeln stehen zwischen den experimentellen Tätigkeiten und den mathematischen Formeln. Mit Hilfe des Pascalschen Dreiecks (bzw. von entsprechenden Baumdiagrammen) lassen sich diese Regeln durch Experiment und ideale Simulation begründen und - wenn man es im Unterricht für möglich hält - in einer 
mathematischen Formel zusammenfassen.

In einer vierten Arbeitsphase lassen sich, bezogen auf das Binomial-Model1, wie es in der Binomialverteilung (durch unterschiedliche Diagramme) und in der Binomialformel (sowohl als allgemeiner Vierteilungsfunktion als auch in beispielbezogener Interpretation) zum Ausdruck kommt, Mathematisierungsprobleme des Galtonbretts diskutieren. Fragen folgender Art könnten beispielsweise behandelt werden:

Ist diese Formel ein angemessenes Model1 für den "Normalzustand" des Galtonbretts?

Was sagt diese Formel aus für das gekippte Galtonbrett?

Daß man auf diese Weise nicht einen angezielten Schlußpunkt in der Begriffsentwicklung erreicht hat, die scheinbar vom Beispiel zur Abstraktion führt, das verhindert das Galtonbrett selbst. So ist z.B. die Binomialverteilung $z u p \neq \frac{1}{2}$ nicht einfach als mathematische Formel:

$$
B_{n}(k, p)=\left(\begin{array}{l}
n \\
k
\end{array}\right) p^{k}(1-p)^{n-k}
$$

eine zureichende stochastische Erklärung für das schräggestellte Galtonbrett. Es fehlen spezifische inhaltliche Begründungen, die zu einem mathematischen Modell führen. Solche Annahmen lassen sich etwa aus der Geometrie des Galtonbretts gewinnen.

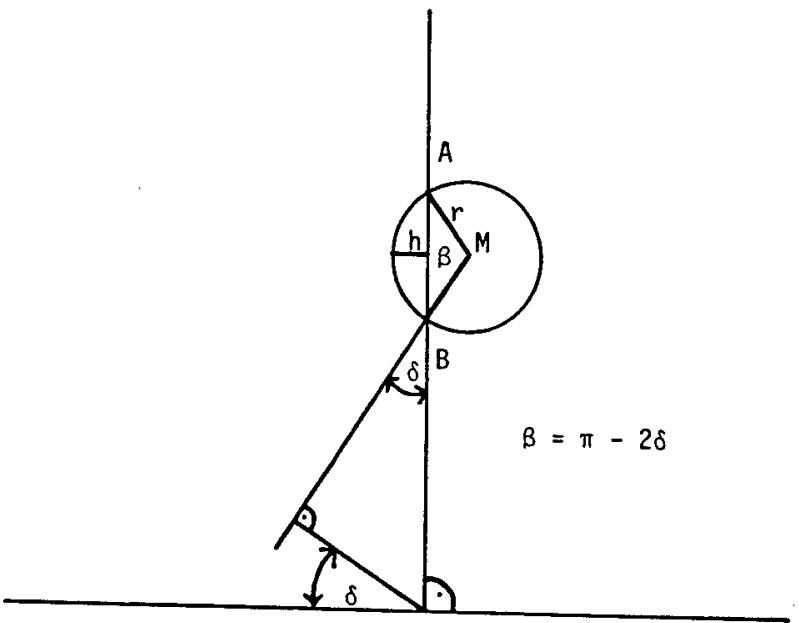

(von HARTEN/STEINBRING, S.103) 
So kann man Wahrscheinlichkeitsannahmen entsprechend den Anteilen der Kugel links und rechts vom Zapfen formulieren (vgl. die Zeichnung, so z.B. Längenabstände, Kreissegmente, Flächenanteile, Volumenanteile). Diese Annahmen führen dann über die Binomialverteilung zu einer Modellbeschreibung, die sich jedoch nur im Vergleich mit experimentellen Ergebnissen als zutreffend erweisen kann.

Wie eng und wie permanent der Gegenstand (hier das Galtonbrett) und die stochastische Modellierung aufeinander bezogen sind, zeigt sich darin, wie z.B. die stochastische Unabhängigkeit mit der konkreten Beschaffenheit des Galtonbretts, mit Anordnung und Form der Zapfen zusammenhängt. Eine genauere Analyse eines Galtonbretts mit zylindrisch geformten Zapfen führt beispielsweise dazu, für zwei aufeinander folgende Stufen keine Unabhängigkeit, sondern (geringfügige) Abhängigkeiten anzunehmen. Diese Annahme verändert die Binomialverteilung in eine sog. "bedingte" Binomialverteilung, eine gegenstandsadäquate Variation, welche die empirisch beobachtete Verteilung dieses Galtonbretts recht gut modelliert. (Eine umfassende Darstellung dieses Sachverhaltes findet sich in (STEINBRING 1985a). Die "bedingte" Binomialverteilung ist eine Begriffsverallgemeinerung, die aus der Analyse des stochastischen Gegenstandes erwachsen ist, nicht durch Abstraktion ("Absehen von bestimmten Eigenschaften äquivalenter Dinge" (SCHREIBER 1980) und auch nicht durch Idealisierung ("Hineinsehen von Eigenschaften, die ein Ding nicht besitzt" (SCHREIBER 1980), sondern durch angemessene mathematische Modellierung von Beziehungen des Gegenstandes, die im realen Experiment wirksam werden.

Nicht die mathematische Formel enthält schon für sich allein die Spezifik der Stochastik. Erst wenn sie als ein Element des gesamten Systems stochastischer Tätigkeiten und Darstellungsmittel aufgefaßt wird, welches den Zusammenhang zwischen konkretem Zufallsphänomen und der stochastischen Modellierung stiftet, ist es möglich, der stochastischen Begrifflichkeit Geltung zu verschaffen. "Das Glücksspiel oder der Würfel oder der Zufallsgenerator oder das Problem der Meßgenauigkeit bestimnen den Begriff von 'Wahrscheinlichkeit', den man in dem jeweiligen Zusammenhang hat, genauso deutlich und genauso stark wie eine entsprechende Definition." (OTTE 1978, S.20)

4. Der mathematische Begriff als Zusammenspiel von experimentellen und theore-

\section{tischen Elementen}

"Die Praxis der Mathematik, besonders in der Schule, wird ... in der Regel durch die Automatisierung, das Algorithmische, wie es die Formel als Berechnungsprozedur ausdrückt, zu einer Identifizierung von Zeichen und Bezeichnetem verführt, oder, wenn man die dreifache Unterscheidung von Begriff, Zeichen und Gegenstand 
macht, die eigentlich notwendig wäre, zu einer Identifizierung von Zeichen und . Gegenstand, bei Vernachlässigung eines davon unabhängigen Begrifflichen." (OTTE 1984, S.19)

Gegenüber diesem Problem vorschneller Identifizierungen von Gegenstand, Zeichen und Begriff erweist sich im Unterricht die Wahrscheinlichkeit als einigermaßen resistent. Eine Gleichsetzung des Gegenstandes mit dem Zeichen wird durch das besondere Verhältnis von konkretem Einzelfall und stochastischem Modell verhindert. Daß die Wahrscheinlichkeit daneben eine eigenständige Rolle spielt, wird durch die Bedeutungsvielfalt dieses Begriffs garantiert.

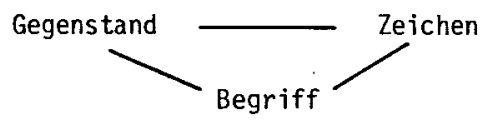

Diese differenzierte Vorgehensweise beim Begriffslernen hat jedoch eine wichtige Voraussetzung: Die duale Struktur des Wahrscheinlichkeitsbegriffs - seine gleichzeitig theoretische und experimentelle Beschaffenheit - darf nicht definitorisch zerstört, d.h. zugunsten einer dieser beiden Seiten festgeschrieben werden. Sonst besteht auch hier die Gefahr, daB das relationale Dreieck "Gegenstand, Zeichen, Begriff" in sich zusammenfällt. Angesichts der Tatsache, daß die Stochastik jedoch nicht einfach als mathematisch-formaler Apparat ihre Spezifik zum Ausdruck bringen kann, ist diese Gefahr vielleicht nicht ganz so groß wie in den anderen Bereichen der Schulmathematik.

Die Notwendigkeit, bewuBt Unterscheidungen zwischen Gegenstand, Zeichen und Begriff zur Entwicklung von Begriffsbedeutungen aufrechtzuerhalten, läßt sich im Stochastikunterricht selbst beobachten. Aus Verständnisproblemen von Schiilern, sowie aus indirekten Hinweisen in Lernprozessen wird sichtbar, wie bei Beschränkung gegenständlicher oder begrifflicher Aspekte auf die mathematischen Zeichen, Kalküle und Strukturen Fragen nach unterliegenden Bedeutungen entstehen ( $\mathrm{vgl}$. STEINBRING 1984 und 1985b).

Die grundlegende Prämisse für einen offen gestalteten Lernprozeß, für eine tatsächliche Begriffsentwicklung im Unterricht - ohne eine voliständige Vorabfestlegung des Begriffs - ist die effektive Berücksichtigung der Komplementarität von theoretischen und experimentellen Aspekten des mathematischen Begriffs ( $\mathrm{vgl}$. AG MATHEMATIKLEHRERBILDUNG, S.205ff). Für die Wahrscheinlichkeit bedeutete dies, in der kontrastierenden Gegenüberstellung beider Kennzeichnungen - experimentell als relative Häufigkeit und theoretisch als relativer Anteil - diesen Begriff zu entwickeln. Durch diese didaktische Maßnahme wird auch den Schülern deutlich, da $B$ der Begriff nicht mit seiner Definition identisch ist. 
Wenn nicht die mathematische Struktur die zentrale Orientierung für den inhaltlichen LernprozeB ist, was steuert dann den Erwerb des mathematischen Begriffs im Unterricht? Die Aneignung und das Verstehen des Begriffs geschieht durch die permanente Entwicklung und den fortschreitenden Gebrauch von vielfältigen Mitteln der Darstellung und Tätigkeit. Das Gesamtsystem der Darstellungs- und Tätigkeitsmittel ermöglicht die oberbrückung der weiten Spanne von konkret-experimentellen und theoretisch-modellhaften Aspekten, wie sie sowohl in der Lernsituation als auch im mathematischen Begriff selbst präsent sind. Für den schulischen Lernproze $\beta$ ist es nötig, die spezifischen Mittel und Bedingungen der "didaktischen Situation" als essentiell für den Begriffserwerb und nicht als nebensächliche Randbedingungen anzusehen. "Anstatt didaktische Situationen lediglich als isolierte Hilfsmittel zur Erzeugung der gewünchten Verhaltensweise zu betrachten, muß man sie ... als Glied einer Kette oder Teil eines Prozesses ansehen, der eine kleine künstliche Begriffsgenese darstellt, wobei diese Prozesse ebenso Ziel wie Mittel des Unterrichts sind" (BROUSSEAU, S.8).

Die Tätigkeits- und Darstellungsmittel in einer didaktischen Situation ermöglichen den Anschluß an die kommunikativen und sozialen Aspekte im allgemeinbildenden Mathematikunterricht. Sie helfen dem Schüler, ein wirksames persönliches Verhältnis zum mathematischen Wissen zu finden. Vor allem die effektive Berücksichtigung konkreter experimenteller Tätigkeiten ist hierfür entscheidend. Der Kontrast zwischen Experiment und Modell, der Vergleich empirischer Ergebnisse mit theoretischen Annahmen und Voraussagen setzt die Begriffsentwicklung in Gang. Diese Entwicklung ist nicht eine Begriffsspezifikation im Rahmen der Mengenlehre, sie ist eine tatsächliche Entwicklung, die neue und dem Schüler vorher unbekannte Einsichten vermittelt, die aus den Widersprüchen von experimentellem Einzelfall und mathematischem Modell den Begriff generiert.

Die Erkenntnis, daß für eine jeweilige Entwicklung des mathematischen Begriffs das System der dazugehörenden Mittel der Tätigkeiten und Darstellungen unerläßlich ist, ermöglicht den Vergleich unter verschiedenen Formen von Begriffsentwicklungen, ohne diese zu identifizieren. Die Begriffsentwicklung in didaktischen Situationen kann z.B. aus der historischen Entwicklung des Begriffs viel lernen, darf jedoch nicht versuchen, diese zu kopieren. Ebenso sind wissenschaftliche oder psychologische Aspekte der Begriffsentwicklung von groBem Interesse, auf sie läßt sich das Begriffslernen jedoch nicht reduzieren. Lernen in didaktischen Situationen bedeutet, da $B$ der mathematische Begriff durch Schüler und Lehrer neu geschaffen werden muB, nicht als Kopie eines unabhängigen wissenschaftlichen Begriffsexemplars, sondern als ein eigenständiger Begriff der Schulmathematik. Dies ermöglichen und garantieren die spezifischen Mittel in der didaktischen Situation. 
0blicherweise orientiert sich die Schulmathematik vornehmlich am Bild der reinen Mathematik. Dies verursacht viele Verkürzungen in der didaktischen Epistemologie und verhindert häufig eine angemessene Darstellung des Begriffslernens als autonomen mathematischen Erkenntnisproze $B$ des Unterrichts. Demgegenüber gestattet das System der Darstellungs- und Tätigkeitsmittel in einer didaktischen Situation, die Begriffsentwicklung im Unterricht als einen "offenen" Proze $B$ zu gestalten. Damit diese "Offenheit" des Lernprozesses - die häufig gefordert wird (WINTER 1983b, VOLLRATH 1984) - grundsätzlich durch inhaltliche Vorkehrungen auch garantiert wird, muß sich die Auffassung von "geschlossener" Mathematik mehr in Richtung "offener" Mathematik wandeln. "Im Gegensatz zur 'geschlossenen Mathematik', bei der abgeschlossene Theorien, sicher funktionierende Algorithmen und möglichst alle Einzelheiten erfassende Modelle im Vordergrund stehen, betont 'offene Mathematik' grundlegende Begriffsbildungen und Darstellungsformen und faßt Mathematik als Angebot an Darstellungs- und Kommunikationsmitteln auf." (FISCHER, S.139) Damit diese Darstellungsmittel kein Selbstzweck werden (wie es häufig die geschlossenen Strukturen und Theorien der Mathematik sind), muB es einen unabhängigen Lern-Gegenstand, eine praktisch wirksame Realität (GOODMAN 1979) neben diesen Mitteln geben, nicht nur als Ausgangspunkt der Entwicklung, sondern diese beständig begleiten und selbst auf der entwickelsten Stufe der Theorie diese korrigieren und weitertreiben.

Auf diese Weise wird Begriffsternen im Unterricht bis zu einem gewissen Grade dem offenen, schöpferischen Forschungsprozeß in der Wissenschaft vergleichbar. Sowohl die Schulmathematik als auch die wissenschaftliche Mathematik basieren nicht auf bloßen formalen Strukturen; beide bedürfen angemessener Mittel und Darstellungen. "... die Objektivierung des mathematischen Wissens, sein Status als wahres und objektives Wissen (ist) nur zu sichern bezogen auf das Universum 'aller' denkbaren Mittel und aller möglichen Darstellungen. Die Frage nach der Gegenständlichkeit der Mathematik ist nur auf der Grundlage 'aller' möglichen Zugangsweisen zum mathematischen Wissen formulierbar." (OTTE 1984, S.II/III)

Auf dieser Grundlage lassen sich Schulmathematik und wissenschaftliche Mathematik aber auch voneinander unterscheiden und abgrenzen. Die mathematischen Erkenntnismittel und Tätigkeiten für Schüler im allgemeinbildenden Unterricht sind allgemeiner, grundlegender, vielfältiger und uneinheitlicher als die speziellen und universellen Techniken, wie sie in der reinen Mathematik entwickelt wurden. Während sich in der Folge der Arbeitsteilung in der Wissenschaft, die allein in der Mathematik zu einer uniberschaubaren Spezialisierungsvielfalt geführt hat, eine disziplinäre Reinheit von Methoden und Mitteln durchgesetzt hat, muB der Mathematikunterricht mit seinem Mittelsystem die Distanz zwischen Alltagswissen und mathematischem Wissen überbrücken. Schulmathematik darf nicht 
nach dem Vorbild wissenschaft]icher Spezialisierung in Einzeldiszipl inen aufgegliedert und untersucht werden. Auf diese Weise ändert sich der epistemologische Status der Schulmathematik entscheidend: Mathematisches Wissen im Unterricht ist kein professionelles und disziplinäres Wissen; Schulmathematik ist al1gemeines und außermathematische Begründungen umfassendes Wissen. Schulmathematik ist nicht unabhängig und universe11, sondern lokal und beispielhaft. Für die Schulmathematik ist die didaktische Situation zusammen mit ihrem Mittelsystem in ähnlicher Weise ein Referenzrahmen, der ihre spezifische Bedeutung zum Ausdruck bringt, wie dies für den Wahrscheinlichkeitsbegriff die verschiedenen spezifischen Referenzrahmen leisten. In der Frage um den Charakter von Schulmathematik stellt sich somit nicht die (wissenschaftliche) Alternative von reiner zu angewandter Mathematik. Für den Unterricht ist das Verhältnis von experimenteller zu theoretischer Mathematik fruchtbarer, welches im Zusammenwirken dieser beiden Pole dif Schulmathematik zu einem besonderen Typ anwendungsorientierter Mathematik macht.

Entsprechend dieser didaktischen Epistemologie müßten für die Begriffe der Schulmathematik Entwicklungsrahmen erarbeitet, d.h. entsprechende Systeme von Darstellungs- und Tätigkeitsmitteln konstruiert werden, die das Zusammenwirken experimenteller und theoretischer Elemente grundsätzlich erfordern. Die Erprobung solcher Begriffssysteme in didaktischen Situationen ist Ausgangspunkt für die Herstellung didaktischer Textmaterialien (für Lehrer und Schüler), für die Kooperation mit Schulpraktikern, für psychologische, soziale und pädagogische Untersuchungen und für vieles mehr. Die Kennzeichnung des mathematischen Wissens als Bestandteil der allgemeinen menschlichen Erkenntnistätigkeit ist hierfür die Voraussetzung. Auf diese Weise wird mathematisches Wissen auch zu einem wirklichen und wichtigen Bezugselement im Gesamtsystem der Mathematikdidaktik.

Dabei ist zu beachten, daß didaktische Analysen zum mathematischen Begriff selbst theoretische Begrifflichkeiten entwickeln und in der Analyse anwenden. Vorschläge zum Lernen und Lehren von Begriffen können nicht vorab eindeutig fixiert sein, weder auf curricularer Ebene, noch in Schemata der Begriffsklassifikationen oder in Operations-bzw. Handlungssystemen. Es handelt sich um theoretische Rahmenkonzepte, die keine spezifischen Begriffsbedeutungen vorgeben, sondern wichtige Beziehungen im Proze $B$ des Wissenserwerbs identifizieren, welche kontextbezogene und unterrichtlich vermittelte Bedeutungsentwicklungen herbeiführen und unterstuitzen sollen. In der aktiven Auseinandersetzung mit den mathematischen Lern-Gegenständen konstituieren sich faktische Begriffsbedeutungen im LernprozeB. 


\section{Literatur}

AG MATHEMATIKLEHRERBILDUNG: Perspektiven für die Ausbildung des Mathematiklehrers. Köln 1981

ANDELFINGER, B.: Didaktischer Informationsdienst Mathematik. Thema: Arithmetik, Algebra und Funktionen. Soest 1985

BIEHLER, R.: Explorative Datenanalyse - Eine Untersuchung aus der Perspektive einer deskriptiv-empirischen Wissenschaftstheorie. Materialen und Studien, Bd. 24, IDM Bielefeld, 1982

BIEHLER, R.: Graphische Darstellungen. Occasional Paper 50, IDM Bielefeld 1984 (erscheint in: math.did. 8, 1985a)

BIEHLER, R.: Die Renaissance graphischer Methoden in der angewandten Statistik, Occasional Paper 65, IDM Bielefeld 1985b

Brousseau, G.: Tendances originales de la recherche en didactiques des mathématiques en France. Manuskript, Bordeaux 1982

DINGES, H.: Zum Umgang mit Aussagen in der Stochastik. Manuskript. Frankfurt 1977

DINGES, H.: Zum Wahrscheintichkeitsbegriff für die Schule, in: Dörfler, W./Fischer, R. (eds.): Stochastik im Schulunterricht. Wien 1981, 49-61

DINGES, H.: Einheitlichkeit, Wirklichkeitsbezug und Kommunizierbarkeit der $\mathrm{Ma}-$ thematik, in: Aktuelle Probleme und Entwicklungstendenzen der Mathematik. Deubner Texte. Leipzig 1982, 83-112

DORFLER, W.: Actions as a Means for Acquiring Mathematical Concepts, in: Proc. 8th Int. Conf. Psychology of Mathematics Education. Sydney 1984a, 172-180

DORFLER, W.: Verallgemeinern als zentrale mathematische Fähigkeit, in: JMD, 4 , $1984 \mathrm{~b}, 239-264$

DORFLER, W.: Das Verhältnis mathematischer Operationen und gegenständlicher Handlungen, erscheint in: Grundfragen der Entwicklung mathematischer Fähigkeiten. Köln 1985

FELLER, W.: An Introduction to Probability Theory and its Applications. Vol.I. New York 1968

FINE, T.L.: Theories of Probability. New York 1973

FISCHER, R.: Offene Mathematik und Visualisierung, in: math.did. $3 / 4$ (1984), $139-160$

FREUDENTHAL, H.: The Implicit Philosophy of Mathematics Today, in: Klibansky, R. (ed.): Contemporary Philosophy, I, Logic and Foundations of Mathematics. Firenze 1968, 342-368

GOODMAN, N.: Mathematics as an Objective Science, in: AMM, vol. 86 (1979), 540-51 (deutsche Obersetzung von G. Seib, IDM Bielefeld)

HARTEN, G.v./STEINBRING, H.: Stochastik in der Sekundarstufe I. Köln 1984 JAGER, J./SCHUPP, H.: Stochastik in der Hauptschule. Paderborn 1983 
JAHNKE, H.N.: Zum Verhältnis von Wissensentwicklung und Begründung in der Mathematik - Beweisen als didaktisches Problem. Materialien und Studien Bd. 10, IDM Bielefeld 1978

JAHNKE, H.N.: Zum Verhältnis von Bildung und wissenschaftlichem Denken am Beispiel der Mathematik. Eine Kontroverse um den mathematischen Lehrplan der preußischen Gymnasien 1829-30 und ihr methodologischer Kontext, in: Bekemeier, $B$. et al (Hrsg.): Wissenschaft und Bildung im frühen 19. Jahrhundert I, Materialien und Studien, Bd. 27, IDM Bielefeld 1982, 1-225

JAHNKE, H.N.: Anschauung und Begründung in der Schulmathematik. Manuskript. Bielefeld 1984

KAISER, G.: Zur Realisierbarkeit von Zielen eines anwendungsorientierten Mathematikunterrichts, in: math.did., 7 (1984), 71-86

KOLMOGOROFF, A.: Grundbegriffe der Wahrscheinlichkeitsrechnung. Berl in 1933

LEPPIG, M.: Vorüberlegungen zum Begriffslernen, in: MU, 1 (1985), 63-74

OTTE, M.: Einige grundsätzliche oberlegungen zum Lehrbuch im Mathematikunterricht. Manuskript. Bielefeld 1978

OTTE, M.: Fachdidaktik als Wissenschaft, Occasional Paper 20, IDM Bielefeld 1982a

OTTE, M.: Zum Problem des Lehrplans und der Lehrplanentwicklung in der Sekundarstufe I, in: Vonhoff, H.-P. (Hrsg.): Bericht zur Lehrplankonferenz Mathematik Sekundarstufe I, Frankfurt 1982, 13-24

OTTE, M.: Was ist Mathematik? Occasional Paper 43, IDM Bielefeld, März 1984

SACKOV, J.V.: Wahrscheinlichkeit und Struktur. Berlin 1978

SCHREIBER, A.: Idealisierungsprozesse - ihr logisches Verständnis und ihre didaktische Funktion, in: JMD, 1/2 (1980), 42-61

SCHUPP, H.: Zum Verhältnis statischer und wahrscheinlichkeitstheoretischer Komponenten im Stochastik-Unterricht der Sekundarstufe I, in: JMD, 3/4 (1982), 207-226

SCHUPP, H.: Sinnvoller Stochastik-Unterricht in der Sekundarstufe I, in: did. math., $3 / 4(1984), 235-243$

STEINBRING, H.: Zur Entwicklung des Wahrscheinlichkeitsbegriffs - Das Anwendungsproblem in der Wahrscheinlichkeitstheorie aus didaktischer Sicht. Materialien und Studien Bd. 18, IDM Bielefeld 1980a

STEINBRING, H.: Zur Entwicklung des Wahrscheinlichkeitsbegriffs - Bernoullis Theorem aus didaktischer Sicht, in: JMD, 3 (1980b), 115-141

STEINBRING, H.: Dimensionen des Verhältnisbegriffs im Stochastikunterricht. Manuskript. Bielefeld 1984 (erscheint in math.did. 1985)

STEINBRING, H.: Experimente mit dem Galtonbrett - Wie sich die Binomialverteilung abhängig von den Versuchsbedingungen ändert. Manuskript. Bielefeld 1985a (erscheint in: mathematik lehren, 12, 1985) 
STEINBRING, H.: Zur Behandlung stochastischer Grundbegriffe im Unterricht - Eine vergleichende Falluntersuchung zwischen Grund- und Erweiterungskurs einer 7. Klasse. Manuskript. Bielefeld 1985b (Kurzfassung erscheint in: Beiträge zum MU, 1985)

STEINER, H.-G.: Theory of Mathematics Education (TME) - An Introductory Talk, in: Steiner, H.-G., Balacheff, N. et al.: Theory of Mathematics Education (TME), Occasional Paper 54, IDM Bielefeld 1984, 16-32

THOM, R.: Modern mathematics: does it exist? in: Howson, A.G. (ed.): Developments in Mathematical Education, Cambridge 1973, 194-209

VOLLRATH, H.-J.: Methodik des Begriffslehrens im Mathematikunterricht. Stuttgart 1984

WINKELMANN, B.: Kritische Anmerkungen zu R. Thom: Moderne Mathematik: Gibt es das?, in: DdM, 1 (1973), 264-274

WINTER, H.: Zur Problematik des Beweisbedürnisses, in: JMD, 1 (1983a), 59-95

WINTER, H.: Ober die Entfaltung begrifflichen Denkens im Mathematikunterricht, in: JMD, 3 (1983b), 175-204

Dr. Heinz Steinbring

Institut für Didaktik der Mathematik

Universität Bielefeld

Postfach 8640

4800 Bielefeld 1 